\title{
OBSTRUCTION THEORY FOR OBJECTS IN ABELIAN AND DERIVED CATEGORIES
}

\author{
WENDY T. LOWEN*
}

\begin{abstract}
In this paper we develop the obstruction theory for lifting complexes, up to quasi-isomorphism, to derived categories of flat nilpotent deformations of abelian categories. As a particular case we also obtain the corresponding obstruction theory for lifting of objects in terms of Yoneda Extgroups. In appendix we prove the existence of miniversal derived deformations of complexes.
\end{abstract}

\section{INTRODUCTION}

Complete families of non-commutative deformations of projective planes, quadrics and more generally Hirzebruch surfaces where constructed in [4, 14, 15] using adhoc deformation theoretic arguments. In order to provide a firmer foundation for these constructions we developed in [9, 10, a deformation theory for abelian categories which generalizes the deformation theory of (module categories over) algebras.

The arguments in [4, 14, 15] are based on the intuition that exceptional objects [3] should lift to any deformation. In the current paper we will justify this assumption by developing an obstruction theory for the lifting of objects (and complexes) to deformations of an abelian category.

Let us first summarize the deformation theory of abelian categories. Assume that $R \longrightarrow R_{0}$ is a surjective ringmap with nilpotent kernel between coherent, commutative rings ${ }^{1}$. A deformation of an $R_{0}$-linear abelian category $\mathcal{C}_{0}$ along $R \longrightarrow$ $R_{0}$ is an $R$-linear functor $\mathcal{C}_{0} \longrightarrow \mathcal{C}$ inducing an equivalence $\mathcal{C}_{0} \cong \mathcal{C}_{R_{0}}$ where $\mathcal{C}_{R_{0}} \subset \mathcal{C}$ is the full subcategory of $R_{0}$-objects, i.e. objects with an $R_{0}$-structure compatible with the $R$-structure [9, Def.5.2, §4]. In general such deformations can be very wild but we show in loc. cit. that by restricting to (appropriately defined) flat deformations the theory becomes controllable.

The definition of flatness for an abelian $R$-linear category is somewhat involved [9. Def.3.2] but for a category with enough injectives it amounts to requiring that injectives are $R$-coflat, i.e. $R$-flat in the dual category [1. An $R_{0}$-algebra $A_{0}$ is flat if and only if $\operatorname{Mod}\left(A_{0}\right)$ is flat, and flat $R$-deformations of $\operatorname{Mod}\left(A_{0}\right)$ correspond precisely to flat $R$-deformations of $A_{0}[9$.

In this paper we will study the problem of lifting objects along the functor $\operatorname{Hom}_{R}\left(R_{0},-\right): \mathcal{C} \longrightarrow \mathcal{C}_{0}$ for a deformation $\mathcal{C}_{0} \longrightarrow \mathcal{C}$ and similarly the problem of lifting objects in the correspoding derived categories along the functor $\mathrm{RHom}_{R}\left(R_{0},-\right)$. By dualizing one obtains lifting properties for the (perhaps more familiar) functors $R_{0} \otimes_{R}-$ and $R_{0} \stackrel{\mathrm{L}}{\mathrm{Q}}_{R}-$. We leave the explicit formulations of these dual versions to the reader. There is a parallel obstruction theory for lifting maps which is contained in the body of the paper, but which for brevity we will not formulate in this introduction.

\footnotetext{
*aspirant at the FWO.

${ }^{1}$ In applications $R$ and $R_{0}$ will probably be artinian local rings but the added generality we allow incurs very little cost
} 
Consider surjective ringmaps between coherent, commutative rings

$$
\bar{R} \longrightarrow R \longrightarrow R_{0}
$$

with $\operatorname{Ker}\left(\bar{R} \longrightarrow R_{0}\right)=I, \operatorname{Ker}(\bar{R} \longrightarrow R)=J$ and $I J=0$. Consider flat abelian deformations $\overline{\mathcal{C}} \longleftarrow \mathcal{C} \longleftarrow \mathcal{C}_{0}$ along these ring maps along with their adjoints

$$
\operatorname{Hom}_{\bar{R}}(R,-): \overline{\mathcal{C}} \longrightarrow \mathcal{C} \text { and } \operatorname{Hom}_{R}\left(R_{0},-\right): \mathcal{C} \longrightarrow \mathcal{C}_{0}
$$

For a functor $F$ and an object $C$ in the codomain of $F, L_{F}(C)$ denotes the natural groupoid of lifts of $C$ along $F$ (Definition 3.1).

We prove the following obstruction theory for lifting coflat objects along the restricted functor

$$
\operatorname{Hom}_{\bar{R}}(R,-): \operatorname{Cof}(\overline{\mathcal{C}}) \longrightarrow \operatorname{Cof}(\mathcal{C})
$$

where $\operatorname{Cof}(-)$ denotes the full subcategory of coflat objects.

Theorem A. Consider a lift $C$ of $C_{0}$ along $\operatorname{Hom}_{R}\left(R_{0},-\right)$.

(1) There is an obstruction

$$
o(C) \in \operatorname{Ext}_{\mathcal{C}_{0}}^{2}\left(R \operatorname{Hom}_{R_{0}}\left(J, C_{0}\right), C_{0}\right)
$$

with

$$
o(C)=0 \Longleftrightarrow L_{\mathrm{Hom}_{\bar{R}}(R,-)}(C) \neq \varnothing .
$$

(2) If $o(C)=0$, then $\operatorname{Sk}\left(L_{\mathrm{Hom}_{\bar{R}}(R,-)}(C)\right)$ is affine over

$$
\operatorname{Ext}_{\mathcal{C}_{0}}^{1}\left(R \operatorname{Hom}_{R_{0}}\left(J, C_{0}\right), C_{0}\right) .
$$

The previous result generalizes the classical obstuction theory for lifting along $\operatorname{Hom}_{\bar{R}}(R,-): \operatorname{Mod}(\bar{A}) \longrightarrow \operatorname{Mod}\left(R \otimes_{\bar{R}} \bar{A}\right)$ for an $\bar{R}$-algebra $\bar{A}$. [8]. Note that as expected when $R_{0}$ is a field, we obtain obstructions purely in terms of the Yoneda Ext-groups $\operatorname{Ext}_{\mathcal{C}_{0}}^{i}\left(C_{0}, C_{0}\right)$.

Theorem $\mathrm{A}$ is closely related to our main Theorem B below (which is contained in Theorem 6.10 in the body of the paper). Theorem B gives the obstruction theory for lifting along the restricted derived functor

$$
R \operatorname{Hom}_{\bar{R}}(R,-): D_{\mathrm{fcd}}^{b}(\overline{\mathcal{C}}) \longrightarrow D_{\mathrm{fcd}}^{b}(\mathcal{C}) .
$$

Here "fcd" means that we restrict to objects of finite coflat dimension (Definition 6.8). The dual of this condition is finite Tor-dimension (ftd), as considered for example in [5].

Theorem B. Consider a lift $C^{\cdot}$ of $C_{0}$ along $R \operatorname{Hom}_{R}\left(R_{0},-\right)$.

(1) There is an obstruction

$$
o\left(C^{\cdot}\right) \in \operatorname{Ext}_{\mathcal{C}_{0}}^{2}\left(R \operatorname{Hom}_{R_{0}}\left(J, C_{0}^{\cdot}\right), C_{0}^{\cdot}\right)
$$

with

$$
o\left(C^{\cdot}\right)=0 \Longleftrightarrow L_{R \operatorname{Hom}_{\bar{R}}(R,-)}\left(C^{\cdot}\right) \neq \varnothing .
$$

(2) If $o\left(C^{\cdot}\right)=0$, then $\operatorname{Sk}\left(L_{R \operatorname{Hom}_{\bar{R}}(R,-)}\left(C^{\cdot}\right)\right)$ is affine over

$$
\operatorname{Ext}_{\mathcal{C}_{0}}^{1}\left(R \operatorname{Hom}_{R_{0}}\left(J, C_{0}^{\cdot}\right), C_{0}^{\cdot}\right) .
$$

Our approach for proving Theorem $\mathrm{B}$ is to look at the functor

$$
K\left(\operatorname{Hom}_{\bar{R}}(R,-)\right): K(\operatorname{Inj}(\overline{\mathcal{C}})) \longrightarrow K(\operatorname{Inj}(\mathcal{C})
$$

between homotopy categories for a deformation with enough injectives, which leads to the problem of naively deforming differentials and cochain maps to fixed graded lifts of complexes. A detailed obstruction theory for this problem (Theorem 3.8) is worked out in section $\$ 3$ for a full additive functor $F: \overline{\mathfrak{c}} \longrightarrow \mathfrak{c}$ with $(\operatorname{Ker}(F))^{2}=0$ (3.2 (4) ) between additive categories. For such a functor, we prove a "crude lifting 
lemma" inspired by the Crude Perturbation Lemma in [11, which implies that every lift of a complex along

$$
K(F): K(\overline{\mathfrak{c}}) \longrightarrow K(\mathfrak{c})
$$

is homotopy equivalent to a lift of its differential to a fixed graded lift. If $F$ is essentially surjective, this leads to the obstruction theory for such along $K(F)$ (Theorem 4.1). Our main example of a functor $F$ with the indicated properties is a linear deformation along $\bar{R} \longrightarrow R$, i.e a $\bar{R}$-linear functor $\overline{\mathfrak{c}} \longrightarrow \mathfrak{c}$ inducing $R \otimes_{\bar{R}} \overline{\mathfrak{c}} \cong \mathfrak{c}$. Here $R \otimes_{\bar{R}} \overline{\mathfrak{c}}$ is obtained from $\overline{\mathfrak{c}}$ by tensoring the hom-sets with $R$.

Consider flat linear deformations

$$
\overline{\mathfrak{c}} \underset{F}{\longrightarrow} \mathfrak{c} \underset{(-)_{0}}{\longrightarrow} \mathfrak{c}_{0}
$$

along $\bar{R} \longrightarrow R \longrightarrow R_{0}$ (here flat means that the hom-sets are flat modules). In Theorem [5.2 we show that the lifts of a complex $C \in K(\mathfrak{c})$ along $K(F)$ are governed by the complex

$$
J \otimes_{R_{0}} \operatorname{Hom}_{\mathfrak{c}_{0}}\left(C_{0}, C_{0}^{\cdot}\right) .
$$

Since for a flat abelian deformation $\overline{\mathcal{C}} \longleftarrow \mathcal{C}$ with enough injectives, $\operatorname{Hom}_{\bar{R}}(R,-)$ : $\operatorname{Inj}(\overline{\mathcal{C}}) \longrightarrow \operatorname{lnj}(\mathcal{C})$ defines a flat linear deformation (Proposition 5.5), the complex (3) for (11) translates into

$$
R \operatorname{Hom}_{\mathcal{C}_{0}}\left(R \operatorname{Hom}_{R_{0}}\left(J, C_{0}\right), C_{0}\right),
$$

which is the complex behind Theorem $\mathrm{B}$

For completeness, we prove the existence of miniversal homotopy and derived deformations of complexes (when we consider trivial linear or abelian deformations of categories) in Appendix, using Schlessingers conditions [12].

To the best of the author's knowledge Theorems A and B (and their generalizations to maps stated below) have not been formulated in the current generality before. However some particular cases are certainly known. For Theorem A we have already mentioned module categories [8]. The case of coherent sheaves over algebraic varieties is also standard (see for example [16]). First order deformations of an object (for a trivial deformation of an abelian category) were classified in [1]. Theorem B was proved by Inaba for the derived category of coherent sheaves over a projective variety [7]. Related results for the derived category of a profinite group are stated in [2].

The author wishes to thank Michel Van den Bergh for suggesting the use of injective resolutions and for several interesting discussions.

\section{NotATIONS AND PRELIMINARIES ON COCHAIN COMPLEXES}

Let $\mathcal{C}$ be a linear category, i.e. a category enriched over some module category. We have the graded category $G(\mathcal{C})=$ Fun $(\mathbb{Z}, \mathcal{C})$ whose objects are denoted by $C$ and are called graded objects. For $C^{\cdot} \in G(\mathcal{C}), C^{\cdot}[n]$ denotes the shifted graded object with $C[n]^{i}=C^{i+n}$. A morphism $C \longrightarrow D^{\cdot}[n]$ is called a graded map of degree $n$ from $C$ to $D$. The composition of a graded map of degree $n$ with a graded map of degee $m$ is a graded map of degree $n+m$. A graded map of degree 1 from $C$ to $C$. will be called a pre-differential on $C$. A differential on $C \cdot$ is a pre-differential $d$ with $d^{2}=0$. For graded objects $C^{\cdot}, D^{\prime} \in G(\mathcal{C})$, we define a graded abelian group $\operatorname{Hom}^{*}\left(C^{\cdot}, D^{*}\right)$ by $\operatorname{Hom}^{n}\left(C^{*}, D^{*}\right)=G(\mathcal{C})\left(C^{\cdot}, D^{\cdot}[n]\right)$. For pre-differentials $d_{C}$ on $C^{\text {. }}$ and $d_{D}$ on $D^{\prime}$, we define the pre-differential $\delta=\delta_{d_{C}, d_{D}}$ on $\operatorname{Hom}\left(C^{\cdot}, D^{*}\right)$ by

$$
\delta^{n}(f)=d_{D} f-(-1)^{n} f d_{C} .
$$


If $d_{C}=d_{D}, \delta$ turns $\operatorname{Hom}\left(C^{\cdot}, C^{\cdot}\right)$ into a cDG-algebra [13]. If $d_{C}$ and $d_{D}$ are differentials, then so is $\delta$. In this case, if $d_{C}=d_{D}$, Hom $\left(C^{*}, C^{\cdot}\right)$ becomes a DGalgebra. A pre-complex $\left(C^{*}, d\right)$ is a graded object $C^{\cdot}$ endowed with a pre-differential $d$, if $d$ is a differential then $\left(C^{\cdot}, d\right)$ is called a (cochain) complex. A cochain map of degree $n$ between pre-complexes $\left(C^{\cdot}, d_{C}\right) \longrightarrow\left(D^{*}, d_{D}\right)$ is a graded map $f \in$ $\operatorname{Hom}^{n}\left(C^{\cdot}, D^{\cdot}\right)$ with $\delta(f)=0$. For graded maps $f, g \in \operatorname{Hom}^{n}\left(C^{\cdot}, D^{\cdot}\right)$, a homotopy $H: f \longrightarrow g$ is a graded map $H \in \operatorname{Hom}^{n-1}\left(C^{\cdot}, D^{\cdot}\right)$ with $\delta(H)=g-f$. Precomplexes, cochain maps and homotopies constitute a bicategory $P(\mathcal{C})$ in which the complexes form a full bisubcategory $C(\mathcal{C})$. The homotopy category $K(\mathcal{C})$ is obtained from $C(\mathcal{C})$ by considering cochain maps up to homotopy. Restricting to bounded below complexes yields the category $K^{+}(\mathcal{C})$. If $\mathcal{C}$ is an abelian category, there is a functor $C(\mathcal{C}) \longrightarrow G(\mathcal{C}): C \cdot \longmapsto H \cdot C$ mapping a cochain complex to its graded homology object. Cochain maps which are mapped onto isomorphisms by this functor are called quasi-isomorphisms. The derived category $D(\mathcal{C})$ is obtained from $C(\mathcal{C})$ by formally inverting all quasi-isomorphisms. Restricting to bounded below or bounded complexes yields the derived categories $D^{+}(\mathcal{C})$ and $D^{b}(\mathcal{C})$ respectively.

\section{LIFTING DIFFERENTIALS AND COCHAIN MAPS}

In 3.2 we develop the obstruction theory for naively lifting differentials and cochain maps along a suitable additive functor, relative to fixed graded lifts (Corollary 3.7 and Theorem 3.8. 3.3 contains some comparison results for the obstructions defined in 3.2 which enable us to prove a "crude homological lifting lemma" (Corollary 3.11) which refers to the Crude Perturbation Lemma in [11. Since the Crude Perturbation Lemma does not immediately apply, we give a proof of Corollary 3.11 in this paper. However, we believe a generalization of [1] to perturbations of "complexes-modulo-a-subcategory" would also capture Corollary 3.11 We start with introducing some terminology.

3.1. Some lift groupoids. In this section we define the various lift groupoids we will use throughout this paper. Let $F: \overline{\mathcal{C}} \longrightarrow \mathcal{C}$ be an arbitrary functor.

Definition 3.1. (1) For an object $C \in \mathcal{C}$, a lift of $C$ along $F$ is an object $\bar{C} \in \overline{\mathcal{C}}$ together with an isomorphism $c: C \cong F(\bar{C})$. A lift $(\bar{C}, c)$ of $C$ will often be denoted simply by $\bar{C}$ or $c$. If $F: \overline{\mathcal{C}} \longrightarrow \mathcal{C}$ is right adjoint to a functor $G: \mathcal{C} \longrightarrow \overline{\mathcal{C}}$, a lift of $C$ along $F$ can be represented by a map $G(C) \longrightarrow \bar{C}$.

(2) For a map $f: C \longrightarrow C^{\prime}$ in $\mathcal{C}$ and lifts $c: C \cong F(\bar{C})$ and $c^{\prime}: C^{\prime} \cong F\left(\overline{C^{\prime}}\right)$ along $F$ of $C$ and $C^{\prime}$ respectively, a lift of $f$ (along $F$ ) relative to $c, c^{\prime}$ is a map $\bar{f}: \bar{C} \longrightarrow \overline{C^{\prime}}$ with $F(\bar{f}) c=c^{\prime} f$. The set of all lifts of $f$ along $F$ relative to $c, c^{\prime}$ will be denoted by

$$
L_{F}\left(f \mid c, c^{\prime}\right) \text {. }
$$

(3) For $C \in \mathcal{C}$, we consider the following groupoid

$$
L_{F}(C) \text { : }
$$

0. Objects of $L_{F}(C)$ are lifts of $C$ along $F$.

1. Morphisms from $(\bar{C}, c: C \cong F(\bar{C}))$ to $\left(\bar{C}^{\prime}, c^{\prime}: C \cong F\left(\bar{C}^{\prime}\right)\right)$ are elements of $L_{F}\left(1_{C}: C \longrightarrow C \mid c, c^{\prime}\right)$ which are isomorphisms in $\overline{\mathcal{C}}$.

Next we define some natural groupoids for lifting complexes and cochain maps "up to homotopy" relative to fixed graded lifts. Let $F: \overline{\mathcal{C}} \longrightarrow \mathcal{C}$ be an additive functor between linear categories. There are induced functors $G(F): G(\overline{\mathcal{C}}) \longrightarrow$ $G(\mathcal{C})$ between the graded categories, $P(F): P(\overline{\mathcal{C}}) \longrightarrow P(\mathcal{C})$ between the categories of pre-complexes and $C(F): C(\overline{\mathcal{C}}) \longrightarrow C(\mathcal{C})$ between the categories of cochain 
complexes. Lifts along $G(F)$ will also be called graded lifts whereas lifts along $C(F)$ will be called lifts.

Definition 3.2. (1) Consider pre-complexes $\left(C^{*}, d_{C}\right),\left(D^{*}, d_{D}\right) \in P(\mathcal{C})$, graded maps $f, g \in \operatorname{Hom}^{n}\left(C^{\cdot}, D^{\cdot}\right)$ and a homotopy $H: f \longrightarrow g$. Suppose we have lifts $\left(\bar{C}, \bar{d}_{C}\right),\left(\bar{D}, \bar{d}_{D}\right) \in P(\overline{\mathcal{C}})$ along $P(F)$ and graded lifts $\bar{f}, \bar{g}$. A graded lift of $H$ (along $F$ ) relative to $\bar{d}_{C}, \bar{d}_{D}, \bar{f}, \bar{g}$ is a graded lift $\bar{H}$ of $H$ which is a homotopy $\bar{H}: \bar{f} \longrightarrow \bar{g}$. We consider the following groupoid

$$
L_{F}\left(H \mid \bar{d}_{C}, \bar{d}_{D}, \bar{f}, \bar{g}\right):
$$

0 . Objects are graded lifts of $H$ relative to $\bar{d}_{C}, \bar{d}_{D}, \bar{f}, \bar{g}$.

1. Morphisms from $\bar{H}$ to $\bar{H}^{\prime}$ are graded lifts $\overline{0}: \bar{H} \longrightarrow \bar{H}^{\prime}$ of $0: H \longrightarrow H$ relative to $\bar{d}_{C}, \bar{d}_{D}, \bar{H}, \bar{H}^{\prime}$.

(2) Consider cochain complexes $\left(C^{\cdot}, d_{C}\right),\left(D^{*}, d_{D}\right) \in C(\mathcal{C})$ and a cochain map $f:\left(C^{\cdot}, d_{C}\right) \longrightarrow\left(D^{\prime}, d_{D}\right)$. For lifts $\left(\bar{C}^{\prime}, \bar{d}_{C}\right),\left(\bar{D}^{*}, \bar{d}_{D}\right) \in C(\overline{\mathcal{C}})$, we put

$$
L_{F}\left(f \mid \bar{d}_{C}, \bar{d}_{D}\right)=L_{F}\left(f \mid \bar{d}_{C}, \bar{d}_{D}, 0,0\right) \text {, i.e. }
$$

0 . Objects are lifts of $f$ along $C(F)$ relative to $\left(\bar{C}, \bar{d}_{C}\right),\left(\bar{D}, \bar{d}_{D}\right)$.

1. Morphisms from $\bar{f}$ to $\bar{f}^{\prime}$ are graded lifts of $0: f \longrightarrow f$ relative to $\bar{d}_{C}, \bar{d}_{D}, \bar{f}, \bar{f}^{\prime}$.

(3) Consider a cochain complex $\left(C^{*}, d\right) \in C(\mathcal{C})$ and a graded lift $\bar{C}$ of $C$.

(a) A graded lift of $d$ along $F$ relative to $\bar{C}$ is an element of $L_{G(F)}(d$ : $\left.C^{\cdot} \longrightarrow C^{\cdot}[1] \mid \bar{C}, \bar{C}[1]\right)$.

(b) A lift of $d$ along $F$ relative to $\bar{C}$ is a graded lift $\bar{d}$ of $d$ with $\bar{d}^{2}=0$, i.e. a differential $\bar{d}$ on $\bar{C}$ making $(\bar{C}, \bar{d})$ into a lift of $\left(C^{\cdot}, d\right)$ along $C(F)$.

We consider the following bigroupoid

$$
L_{F}(d \mid \bar{C}):
$$

0 . Objects (0-cells) are lifts of $d$ relative to $\bar{C}$.

1. Morphisms (1-cells) from $\bar{d}$ to $\bar{d}^{\prime}$ are lifts of $1:\left(C^{\cdot}, d\right) \longrightarrow\left(C^{\cdot}, d\right)$ relative to $(\bar{C}, \bar{d}),\left(\bar{C}, \bar{d}^{\prime}\right)$ which are isomorphisms in $C(\overline{\mathcal{C}})$.

2. 2-cells from $\overline{1}$ to $\overline{1}^{\prime}$ are graded lifts of $0: 1 \longrightarrow 1$ relative to $\bar{d}, \bar{d}^{\prime}, \overline{1}, \overline{1}^{\prime}$.

3.2. Obstruction theory. In this section we give an obstruction theory for the lift groupoids defined in the previous section under certain assumptions on $F$. For the additive functor $F: \overline{\mathcal{C}} \longrightarrow \mathcal{C}$, let $\operatorname{Ker}(F)\left(\right.$ resp. $\left.\operatorname{Ker}(F)^{2}\right)$ be the category-withoutidentities with the same objects as $\overline{\mathcal{C}}$ and containing precisely the $\overline{\mathcal{C}}$-morphisms $f$ with $F(f)=0$ (resp. the compositions of two such morphisms). From now on we will assume that $F$ is full and

$$
\operatorname{Ker}(F)^{2}=0
$$

This has the following important consequence, which generalizes the well known fact for rings:

Proposition 3.3. Suppose $f: C \longrightarrow D$ and $g: D \longrightarrow C$ are inverse isomorphisms in $\mathcal{C}$ and consider lifts $\bar{C}, \bar{D}$ of $C$ and $D$ respectively. For every lift $\bar{f}: \bar{C} \longrightarrow \bar{D}$ of $f$, there exists a lift $\bar{g}: \bar{D} \longrightarrow \bar{C}$ of $g$ such that $\bar{f}$ and $\bar{g}$ are inverse isomorphisms. In particular, $\bar{C}$ and $\bar{D}$ are isomorphic.

Proof. Consider an arbitrary lift $\bar{g}^{\prime}$ of $g$ and suppose $\bar{f} \bar{g}-1=\epsilon \in \operatorname{Ker}(F)$. It suffices to change $\bar{g}^{\prime}$ into $\bar{g}=\bar{g}^{\prime}(1-\epsilon)$.

In particular, the requirements in Definitions 3.1 (3) and 3.2 that morphisms in the lift groupoids are isomorphisms in $\overline{\mathcal{C}}$ and $C(\overline{\mathcal{C}})$ respectively are automatically 
fulfilled. All (graded) lifts will be along $F$, so we will no longer explicitely say so. For legibility, we will suppress $F$ in all our notations.

Remark 3.4. If we are only interested in lifting complexes of objects in a certain subcategory $\mathcal{C}^{\prime} \subset \mathcal{C}$, by restricting the codomain of $F$, it suffices to require that $F$ is full on the closure of $\mathcal{C}^{\prime}$ under isomorphic objects.

Consider pre-complexes $\left(C^{*}, d_{C}\right)$ and $\left(D^{*}, d_{D}\right)$ with fixed graded lifts $\bar{C}$ and $\bar{D}$. We define the pre-complex

$$
(\mathbf{C}, \bar{\delta})=(\mathbf{C}, \bar{\delta})_{d_{C}, d_{D}}
$$

to be the kernel in the exact sequence of pre-complexes

$$
0 \longrightarrow\left(\mathbf{C}^{\prime}, \bar{\delta}\right) \longrightarrow\left(\operatorname{Hom}^{\cdot}(\bar{C}, \bar{D}), \bar{\delta}\right) \longrightarrow\left(\operatorname{Hom}^{\cdot}\left(C^{\cdot}, D^{\cdot}\right), \delta\right) \longrightarrow 0
$$

where $\delta=\delta_{d_{C}, d_{D}}$ and $\bar{\delta}=\delta_{\bar{d}_{C}, \bar{d}_{D}}$ for arbitrary graded lifts $\bar{d}_{C}, \bar{d}_{D}$ of $d_{C}, d_{D}$ respectively (i.e. $\bar{\delta}^{n}(f)=\bar{d}_{D} f-(-1)^{n} f \bar{d}_{C}$, see also $\underline{2}$ ).

Proposition 3.5. $(\mathbf{C}, \bar{\delta})$ is a cochain complex which is independent of the choice of $\bar{d}_{C}, \bar{d}_{D}$.

Proof. For $f \in \mathbf{C}$, we have $\overline{\delta \delta}(f)=\bar{d}_{D}^{2} f-f \bar{d}_{C}^{2}$. Since $f, \bar{d}_{C}^{2}$ and $\bar{d}_{D}^{2}$ belong to $\operatorname{Ker}(G(F))$, the expression equals zero by (4). Next, other graded lifts of $d_{C}$ and $d_{D}$ can be written as $\bar{d}_{C}+\partial_{C}$ and $\bar{d}_{D}+\partial_{D}$ for $\partial_{C}, \partial_{D} \in \operatorname{Ker}(G(F))$. Hence, for $f \in \mathbf{C}$, we have $\delta_{\bar{d}_{C}+\partial_{C}, \bar{d}_{D}+\partial_{D}}(f)=\delta_{\bar{d}_{C}, \bar{d}_{D}}(f)+\partial_{D} f-(-1)^{n} f \partial_{C}$ and the last two terms equal zero by (4).

The following theorem gives the obstruction theory for lifting homotopies. It has the obstruction theory for lifting cochain maps as an immediate corollary (Corollary 3.77). For any category $\mathcal{D}$, the skeleton $\operatorname{Sk}(\mathcal{D})$ of $\mathcal{D}$ is the class of all isomorphism classes of $\mathcal{D}$-objects.

Theorem 3.6. Consider the following data in $\mathcal{C}$ :

- Pre-complexes $\left(C^{\cdot}, d_{C}\right)$ and $\left(D^{*}, d_{D}\right)$.

- Graded maps $f, g: C \longrightarrow D$ of degree $n$.

- A homotopy $H: f \longrightarrow g$.

Suppose we have fixed lifts $\left(\bar{C}, \bar{d}_{C}\right)$ and $\left(\bar{D}, \bar{d}_{D}\right)$ along $P(F)$ of $\left(C^{\cdot}, d_{C}\right)$ and $\left(D^{*}, d_{D}\right)$ respectively. On $\operatorname{Hom}(\bar{C}, \bar{D})$, put $\bar{\delta}=\delta_{\bar{d}_{C}, \bar{d}_{D}}$. Put

$$
\mathbf{C}=(\mathbf{C}, \bar{\delta})_{d_{C}, d_{D}} .
$$

Suppose we have graded lifts $\bar{f}, \bar{g}, \bar{H}$ of $f, g, H$ respectively with $\bar{\delta}(\bar{f})=\bar{\delta}(\bar{g})$. Put $L(H)=L\left(H \mid \bar{d}_{C}, \bar{d}_{D}, \bar{f}, \bar{g}\right)$.

(1) There is an obstruction

$$
o_{n}(H)=o_{n}\left(H \mid \bar{d}_{C}, \bar{d}_{D}, \bar{f}, \bar{g}\right)=[\bar{g}-\bar{f}-\bar{\delta}(\bar{H})] \in H^{n} \mathbf{C}
$$

with

$$
o_{n}(H)=0 \Longleftrightarrow L(H) \neq \varnothing .
$$

(2) If $o_{n}(H)=0$, the map

$$
v_{n-1}:|L(H)|^{2} \rightarrow H^{n-1} \mathbf{C}:\left(\bar{H}, \bar{H}^{\prime}\right) \mapsto\left[\bar{H}^{\prime}-\bar{H}\right]
$$

satisfies

$$
v_{n-1}\left(\bar{H}, \bar{H}^{\prime}\right)=0 \Longleftrightarrow[\bar{H}]=\left[\bar{H}^{\prime}\right] \in \operatorname{Sk}(L(H))
$$

and induces an $H^{n-1} \mathbf{C}$-affine structure on $\operatorname{Sk}(L(H))$. 
Proof. Without loss of generality, we assume that $F(\bar{C})=C$ and $F(\bar{D})=D$.

(1) Clearly, $F(\bar{g}-\bar{f}-\bar{\delta}(\bar{H}))=g-f-\delta(H)=0$ and $\bar{\delta}(\bar{g}-\bar{f}-\bar{\delta}(\bar{H}))=$ $\bar{\delta}(\bar{g})-\bar{\delta}(\bar{f})=0$, so $\bar{g}-\bar{f}-\bar{\delta}(\bar{H})$ is in $Z^{n}(\mathbf{C})$. Furthermore, $L\left(H \mid \bar{d}_{C}, \bar{d}_{D}, \bar{f}, \bar{g}\right) \neq \varnothing$ if and only if there exists a $\gamma \in \mathbf{C}^{n-1}$ such that $\bar{H}+\gamma$ is a homotopy $\bar{f} \longrightarrow \bar{g}$ or in other words $\bar{g}-\bar{f}-\bar{\delta}(\bar{H})=\bar{\delta}(\gamma)$ which finishes the proof of (1).

(2) Since $0: \bar{H} \longrightarrow \bar{H}^{\prime}$ is a graded lift of $0: H \longrightarrow H$, by part (1) we have $o_{n-1}\left(0: H \longrightarrow H \mid \bar{d}_{C}, \bar{d}_{D}, \bar{H}, \bar{H}^{\prime}\right)=\left[\bar{H}^{\prime}-\bar{H}\right]$ which proves the first part of (2). Now it is easily seen that

$$
a_{n-1}:|L(H)| \times Z^{n-1} \mathbf{C} \longrightarrow|L(H)|:(\bar{H}, \gamma) \longmapsto \bar{H}+\gamma
$$

defines a strictly transitive action $\tilde{a}_{n-1}: \operatorname{Sk}(L(H)) \times H^{n-1} \mathbf{C} \longrightarrow \operatorname{Sk}(L(H))$ with difference map $\tilde{v}_{n-1}: \operatorname{Sk}(L(H))^{2} \longrightarrow H^{n-1} \mathbf{C}$ induced by $v_{n-1}$.

Corollary 3.7. Consider the following data in $\mathcal{C}$ :

- Pre-complexes $\left(C^{\cdot}, d_{C}\right)$ and $\left(D^{*}, d_{D}\right)$.

- A cochain map $f:\left(C^{\cdot}, d_{C}\right) \longrightarrow\left(D^{*}, d_{D}\right)$ of degree $n$.

Suppose we have fixed lifts $\left(\bar{C}, \bar{d}_{C}\right)$ and $\left(\bar{D}, \bar{d}_{D}\right)$ along $P(F)$ of $\left(C^{\cdot}, d_{C}\right)$ and $\left(D^{\cdot}, d_{D}\right)$ respectively. Put

$$
\mathbf{C}=(\mathbf{C}, \bar{\delta})_{d_{C}, d_{D}}
$$

and put $L(f)=L\left(f \mid \bar{d}_{C}, \bar{d}_{D}\right)$. Suppose we have a graded lift $\bar{f}$ of $f$.

(1) There is an obstruction

$$
o_{n+1}(f)=o_{n+1}\left(f \mid \bar{d}_{C}, \bar{d}_{D}\right)=[\bar{\delta}(\bar{f})] \in H^{n+1} \mathbf{C}
$$

with

$$
o_{n+1}(f)=0 \Longleftrightarrow L(f) \neq \varnothing .
$$

(2) If $o_{n+1}(f)=0$, the map

$$
o_{n}:|L(f)|^{2} \longrightarrow H^{n} \mathbf{C}:\left(\bar{f}, \bar{f}^{\prime}\right) \longmapsto\left[\bar{f}^{\prime}-\bar{f}\right]
$$

satisfies

$$
o_{n}\left(\bar{f}, \bar{f}^{\prime}\right)=0 \Longleftrightarrow[\bar{f}]=\left[\bar{f}^{\prime}\right] \in \operatorname{Sk}(L(f))
$$

and induces an $H^{n} \mathbf{C}$-affine structure on $\operatorname{Sk}(L(f))$.

Proof. This is a special case of Theorem 3.6 since a cochain map $f$ is a homotopy $f: 0 \longrightarrow 0$ and we can lift both zeros to zero.

The following theorem gives the obstruction theory for lifting differentials.

Theorem 3.8. Consider a cochain complex $\left(C^{\cdot}, d\right)$ in $\mathcal{C}$ with a fixed graded lift $\bar{C}$ of $C$. Put

$$
\mathbf{C}=(\mathbf{C}, \bar{\delta})_{d, d}
$$

and put $L(d)=L(d \mid \bar{C})$.

(1) There is an obstruction

$$
o(d)=o(d \mid \bar{C})=\left[\bar{d}^{2}\right] \in H^{2} \mathbf{C}
$$

with

$$
o(d)=0 \Longleftrightarrow L(d) \neq \varnothing .
$$

(2) If $o(d)=0$, the map

$$
v:|L(d)|^{2} \longrightarrow H^{1} \mathbf{C}:\left(\bar{d}, \bar{d}^{\prime}\right) \longmapsto\left[\bar{d}^{\prime}-\bar{d}\right]
$$

satisfies

$$
v\left(\bar{d}, \bar{d}^{\prime}\right)=0 \Longleftrightarrow[\bar{d}]=\left[\bar{d}^{\prime}\right] \in \operatorname{Sk}(L(d))
$$

and induces an $H^{1} \mathbf{C}$-affine structure on $\operatorname{Sk}(L(d))$. 
(3) If $v\left(\bar{d}, \bar{d}^{\prime}\right)=0$, the map

$$
w: L(d)\left(\bar{d}, \bar{d}^{\prime}\right)^{2} \longrightarrow H^{0} \mathbf{C}:\left(\overline{1}, \overline{1}^{\prime}\right) \longmapsto\left[\overline{1}^{\prime}-\overline{1}\right]
$$

satisfies

$$
w\left(\overline{1}, \overline{1}^{\prime}\right)=0 \Longleftrightarrow[\overline{1}]=\left[\overline{1}^{\prime}\right] \in \operatorname{Sk}\left(L(d)\left(\bar{d}, \bar{d}^{\prime}\right)\right)
$$

and induces an $H^{0} \mathbf{C}$-affine structure on $\operatorname{Sk}\left(L(d)\left(\bar{d}, \bar{d}^{\prime}\right)\right)$.

Proof. Without loss of generality, we assume that $F(\bar{C})=C$. On Hom $(\bar{C}, \bar{C})$, we put $\bar{\delta}=\delta_{\bar{d}, \bar{d}}$ for a graded lift $\bar{d}$ of $d$.

(1) Clearly, $F\left(\bar{d}^{2}\right)=d^{2}=0$ and $\bar{\delta}\left(\bar{d}^{2}\right)=\bar{d}^{3}-\bar{d}^{3}=0$, so $\bar{d}^{2}$ is in $Z^{2}(\mathbf{C})$. Furthermore, $L(d \mid \bar{C}) \neq \varnothing$ if and only if there exists a $\partial \in \mathbf{C}^{1}$ such that $\bar{d}+\partial$ is a differential on $\bar{C}$ or in other words $\bar{d}^{2}+\bar{\delta}(\partial)=0$ which finishes the proof of (1).

(2) By Lemma 3.5 the differentials $\delta_{\bar{d}, \bar{d}}$ and $\delta_{\bar{d}, \bar{d}^{\prime}}$ coincide on $\mathbf{C}$. Consequently, since $1: \bar{C} \longrightarrow \bar{C}$ is a graded lift of $1: C \longrightarrow C$, we have $o_{1}(1: C \longrightarrow$ $\left.C \cdot \mid \bar{d}, \bar{d}^{\prime}\right)=\left[\bar{d}^{\prime}-\bar{d}\right]$ by Corollary $3.7(1)$ which proves the first part of (2). Now it is easily seen that

$$
a:|L(d)| \times Z^{1} \mathbf{C} \longrightarrow|L(d)|:(\bar{d}, \partial) \longmapsto \bar{d}+\partial
$$

defines a strictly transitive action $\tilde{a}: \operatorname{Sk}(L(d)) \times H^{1}(\mathbf{C}) \longrightarrow \operatorname{Sk}(L(d))$ with difference map $\tilde{v}: \operatorname{Sk}(L(d))^{2} \longrightarrow H^{1}(\mathbf{C})$ induced by $v$.

(3) This follows from Corollary $3.7(2)$.

3.3. Crude lifting lemma. The main theorem of this section, Theorem [3.9] is entirely inspired by [6. Theorem 1.2], [11, Theorem 3]. However, to apply these results, we would have to start with a homotopy equivalence in $\overline{\mathcal{C}}$ and perturb it with respect to the filtration coming from $F: \overline{\mathcal{C}} \longrightarrow \mathcal{C}$, whereas we merely start with a "homotopy equivalence modulo $F$ " in the first place. In 11, Markl shows that the element $[H g-g K] \in H^{-1} \operatorname{Hom}\left(D^{*}, C^{\cdot}\right)$ is the obstruction against the extension of the given homotopy equivalence to a strong one (11, Definition 1]), for which an Ideal Perturbation Lemma 11] IPL] holds. He also shows that changing $K$ into $K^{\prime}=K+f(H g-g K)$ kills this obstruction [11, Theorem 13], yielding [11, Theorem $3]$ and the Crude Perturbation Lemma.

Theorem 3.9. Consider the following data in $\mathcal{C}$ :

- Cochain complexes $\left(C^{*}, d_{C}\right)$ and $\left(D^{*}, d_{D}\right)$.

- Cochain maps $f: C^{\cdot} \longrightarrow D^{\cdot}$ and $g: D^{\cdot} \longrightarrow C^{\text {. }}$.

- Homotopies $H: g f \longrightarrow 1_{C}$ and $K: f g \longrightarrow 1_{D}$.

Suppose we have fixed graded lifts $\bar{C}$ and $\bar{D}$ of $C$ and $D$ respectively.

(1) Suppose we have $\bar{d}_{D} \in L\left(d_{D} \mid \bar{D}\right)$. There exists a $\bar{d}_{C} \in L\left(d_{C} \mid \bar{C}\right)$ with $o\left(f \mid \bar{d}_{C}, \bar{d}_{D}\right)=0$.

(2) Suppose we have $\bar{d}_{D} \in L\left(d_{D} \mid \bar{D}\right), \bar{d}_{C} \in L\left(d_{C} \mid \bar{C}\right)$ and $\bar{f} \in L\left(f \mid \bar{d}_{C}, \bar{d}_{D}\right)$. If

$$
[H g-g K]=0 \in H^{-1} \operatorname{Hom}\left(D^{\cdot}, C^{\cdot}\right)
$$

then there exist $\bar{g} \in L\left(g \mid \bar{d}_{D}, \bar{d}_{C}\right)$ and homotopies $\bar{H}: \bar{g} \bar{f} \longrightarrow 1$ and $\bar{K}$ : $\bar{f} \bar{g} \longrightarrow 1$ lifting $H$ and $K$.

(3) If we change $K$ into $K^{\prime}=K+f(H g-g K)$, then (6) holds.

Proof. Consider $\bar{d}_{D} \in L\left(d_{D} \mid \bar{D}\right)$ and take graded lifts $\bar{d}_{C}, \bar{f}, \bar{g}, \bar{H}, \bar{K}$ where appropriate. We will abusively denote $\delta_{\bar{d}_{C}, \bar{d}_{C}}, \delta_{\bar{d}_{C}, \bar{d}_{D}}, \delta_{\bar{d}_{D}, \bar{d}_{C}}$ and $\delta_{\bar{d}_{D}, \bar{d}_{D}}$ by $\bar{\delta}$. We will gradually change $\bar{d}_{C}, \bar{f}, \bar{g}, \bar{H}, \bar{K}$ until the required properties hold. At any stage of the proof, we put $\mu_{H}=1-\bar{g} \bar{f}-\bar{\delta}(\bar{H}) \in \operatorname{Ker}(G(F))$ and $\mu_{K}=1-\bar{f} \bar{g}-\bar{\delta}(\bar{K}) \in$ 
$\operatorname{Ker}(G(F))$ for the current graded lifts and we also have $\bar{d}_{C}^{2}, \bar{\delta}(\bar{f})$ and $\bar{\delta}(\bar{g})$ in $\operatorname{Ker}(G(F))$.

First, we will show that $o\left(d_{C} \mid \bar{C}\right)=0$. We have $\overline{\delta \delta}(\bar{f})=\bar{d}_{D}^{2} \bar{f}-\overline{f d}_{C}^{2}=-\overline{f d}_{C}^{2}$ and consequently $\bar{d}_{C}^{2}=\bar{\delta}(\eta)$ for some $\eta$ with $F(\eta)=0$ by Lemma [3.10](2) below. So from now on, we may and will suppose that

$$
\bar{d}_{C}^{2}=0
$$

Next we will change $\bar{d}_{C}$ into $\bar{d}_{C}+\partial_{C}$ for some $\partial_{C}$ with $F\left(\partial_{C}\right)=0=\bar{\delta}\left(\partial_{C}\right)$ in order to make $0=o(f)=o\left(f \mid \bar{d}_{C}+\partial_{C}, \bar{d}_{D}\right)=\left[\bar{\delta}(\bar{f})-\bar{f} \partial_{C}\right]$. By Lemma 3.10 $(1), o(f)=0$ for $\partial_{C}=\bar{g} \bar{\delta}(\bar{f})$. From now on, we may and will suppose that

$$
\bar{\delta}(\bar{f})=0 .
$$

Next we show that this implies $o(g)=o\left(g \mid \bar{d}_{D}, \bar{d}_{C}\right)=0$. Indeed, since $\bar{\delta}\left(\mu_{K}\right)=$ $\bar{\delta}(\bar{f}) \bar{g}+\overline{f \delta}(\bar{g})=\overline{f \delta}(\bar{g}), o(g)=0$ by Lemma 3.10 2 ). From now on, we may and will suppose that

$$
\bar{\delta}(\bar{g})=0 .
$$

Finally we will change $\bar{g}$ into $\bar{g}+\gamma$ in order to make $o(H)=o\left(H \mid \bar{d}_{C}, \bar{d}_{C},(\bar{g}+\right.$ $\gamma) \bar{f}, 1)=0$ and $o(K)=o\left(K \mid \bar{d}_{D}, \bar{d}_{D}, \bar{f}(\bar{g}+\gamma), 1\right)=0$. We have $o(H)=\mu_{H}-\gamma \bar{f}$ and $o(K)=\mu_{K}-\bar{f} \gamma$. By Lemma 3.10 $(2), o(H)=0$ for $\gamma=\mu_{H} \bar{g}+\bar{\delta}(\epsilon)$ where $F(\epsilon)=0$, whereas $o(K)=0$ for $\gamma=\bar{g} \mu_{K}+\bar{\delta}(\epsilon)$ where $F(\epsilon)=0$. Hence $o(H)=0=o(K)$ for $\gamma=\mu_{H} \bar{g}$ provided

$$
0=\left[\mu_{H} \bar{g}-\bar{g} \mu_{K}\right]=[\bar{\delta}(\bar{H} \bar{g}-\bar{g} \bar{K})] .
$$

But by assumption, $\bar{H} \bar{g}-\bar{g} \bar{K}=\bar{\delta}(\bar{z})+\zeta$ with $F(\zeta)=0$, hence $\bar{\delta}(\bar{H} \bar{g}-\bar{g} \bar{K})=\bar{\delta}(\zeta)$ and (7) holds, which finishes the proof.

Lemma 3.10. With the notations of the proof of Theorem 3.9 let $\xi$ be a graded map in $\overline{\mathcal{C}}$ with $F(\xi)=\bar{\delta}(\xi)=0$.

(1) We have that any of $(1-\bar{g} \bar{f}) \xi, \xi(1-\bar{g} \bar{f}),(1-\bar{f} \bar{g}) \xi$ and $\xi(1-\bar{f} \bar{g})$ equals $\bar{\delta}(\epsilon)$ for some $\epsilon$ with $F(\epsilon)=0$.

(2) Suppose either $\bar{f} \xi, \xi \bar{f}, \bar{g} \xi$ or $\xi \bar{g}$ equals $\bar{\delta}(\epsilon)$ for some $\epsilon$ with $F(\epsilon)=0$. Then we have $\xi=\bar{\delta}(\eta)$ for some $\eta$ with $F(\eta)=0$.

Proof. (1) We have $(1-\bar{g} \bar{f}) \xi=\left(\mu_{H}+\bar{\delta}(\bar{H}) \xi=\mu_{H} \xi+\bar{\delta}(\bar{H} \xi)+\bar{H} \delta(\xi)\right.$, in which the first term equals zero since $F\left(\mu_{H}\right)=0=F(\xi)$ and $\operatorname{Ker}(F)^{2}=0$, the last term equals zero since $\bar{\delta}(\xi)=0$, and in the middle term, $F(\bar{H} \xi)=F(\bar{H}) F(\xi)=0$ which proves (1). (2) Suppose $\bar{f} \xi=\bar{\delta}(\epsilon)$ and $F(\epsilon)=0$. By (1), $\xi=\bar{g} \bar{f} \xi+\bar{\delta}(\rho)$ with $F(\rho)=0$ and $\bar{g} \bar{f} \xi=\bar{g} \bar{\delta}(\epsilon)=\bar{\delta}(\bar{g} \epsilon)-\bar{\delta}(\bar{g}) \epsilon$ in which the second term equals zero since $F(\bar{\delta}(\bar{G}))=0=F(\epsilon)$ and in the first term, $F(\bar{g} \epsilon)=0$ which proves $(2)$.

Corollary 3.11 (crude lifting lemma). Consider the following data in $\mathcal{C}$ :

- Cochain complexes $\left(C^{\cdot}, d_{C}\right)$ and $\left(D^{*}, d_{D}\right)$.

- Cochain maps $f: C \cdot C^{\cdot}$ and $g: D^{\cdot} \longrightarrow C^{\cdot}$ homotopy inverse to each other.

Suppose we have fixed graded lifts $\bar{C}$ and $\bar{D}$ of $C \cdot$ and $D$ respectively.

(1) Suppose we have $\bar{d}_{D} \in L\left(d_{D} \mid \bar{D}\right)$. There exists a $\bar{d}_{C} \in L\left(d_{C} \mid \bar{C}\right)$ with $o\left(f \mid \bar{d}_{C}, \bar{d}_{D}\right)=0$.

(2) Suppose we have $\bar{d}_{D} \in L\left(d_{D} \mid \bar{D}\right), \bar{d}_{C} \in L\left(d_{C} \mid \bar{C}\right)$. For every $\bar{f} \in L\left(f \mid \bar{d}_{C}, \bar{d}_{D}\right)$, there exists a $\bar{g} \in L\left(g \mid \bar{d}_{D}, \bar{d}_{C}\right)$ such that $\bar{f}$ and $\bar{g}$ are homotopy inverse to each other. In particular, $\bar{f}$ is a homotopy equivalence.

Proof. This follows from Theorem 3.9 
The following proposition is a similar result for homotopies, showing in particular that the obstructions for lifting homotopies are well-defined up to homotopy.

Proposition 3.12. Consider the following data in $\mathcal{C}$ :

- Cochain complexes $\left(C^{\cdot}, d_{C}\right)$ and $\left(D^{*}, d_{D}\right)$.

- Graded maps $f, g: C \longrightarrow D$ of degree $n$.

- Homotopies $H, K: f \longrightarrow g$.

- A homotopy $\Lambda: H \longrightarrow K$.

Suppose we have fixed lifts $\left(\bar{C}, \bar{d}_{C}\right)$ and $\left(\bar{D}, \bar{d}_{D}\right)$ of $\left(C^{\cdot}, d_{C}\right)$ and $\left(D, d_{D}\right)$ respectively. On $\operatorname{Hom}^{\cdot}\left(C^{\cdot}, D^{\cdot}\right)$, put $\delta=\delta_{d_{C}, d_{D}}$ and on $\operatorname{Hom}(\bar{C}, \bar{D})$, put $\bar{\delta}=\delta_{\bar{d}_{C}, \bar{d}_{D}}$.

(1) Suppose there exist graded lifts $\bar{f}, \bar{g}$ of $f, g$ with $\bar{\delta}(\bar{f})=\bar{\delta}(\bar{g})$. We have

$$
o_{n-1}\left(H \mid \bar{d}_{C}, \bar{d}_{D}, \bar{f}, \bar{g}\right)=o_{n-1}\left(K \mid \bar{d}_{C}, \bar{d}_{D}, \bar{f}, \bar{g}\right)
$$

If these obstructions vanish, then for every $\bar{H} \in L\left(H \mid \bar{d}_{C}, \bar{d}_{D}, \bar{f}, \bar{g}\right)$, there exists a $\bar{K} \in L\left(K \mid \bar{d}_{C}, \bar{d}_{D}, \bar{f}, \bar{g}\right)$ with

$$
o_{n-2}\left(\Lambda \mid \bar{d}_{C}, \bar{d}_{D}, \bar{H}, \bar{K}\right)=0 .
$$

(2) Suppose $f, g$ are cochain maps. We have

$$
o_{n}\left(f \mid \bar{d}_{C}, \bar{d}_{D}\right)=o_{n}\left(g \mid \bar{d}_{C}, \bar{d}_{D}\right)
$$

If these obstructions vanish, then for every $\bar{f} \in L\left(f \mid \bar{d}_{C}, \bar{d}_{D}\right)$ there exists a $\bar{g} \in L\left(g \mid \bar{d}_{C}, \bar{d}_{D}\right)$ with

$$
o_{n-1}\left(H \mid \bar{d}_{C}, \bar{d}_{D}, \bar{f}, \bar{g}\right)=0 .
$$

Proof. (1) We have $\left.o_{n-1}(H)-o_{n-1}(K)=[\bar{\delta}(\bar{K})-\bar{\delta}(\bar{H})]=[\bar{\delta}(\bar{\delta}(\bar{\Lambda})-\beta))\right]$ for some $\beta \in \mathbf{C}^{n-1}$ which proves the first part of (1). For the second part, it suffices to take an arbitrary $\bar{K}^{\prime} \in L\left(K \mid \bar{d}_{C}, \bar{d}_{D}, \bar{f}, \bar{g}\right)$ and put $\bar{K}=\bar{K}^{\prime}-\kappa$ for some $\kappa$ representing $o_{n-2}\left(\Lambda \mid \bar{d}_{C}, \bar{d}_{D}, \bar{H}, \bar{K}^{\prime}\right)$. (2) is a special case of (1).

\section{LifTing IN THE HOMOTOPY CATEGORY}

In this section we will use the results of the previous sections to deduce the obstruction theory for lifting objects and maps along the functor

$$
K(F): K(\overline{\mathcal{C}}) \longrightarrow K(\mathcal{C})
$$

between the homotopy categories for an essentially surjective, full additive functor $F: \overline{\mathcal{C}} \longrightarrow \mathcal{C}$ with $\operatorname{Ker}(F)^{2}=0$.

Theorem 4.1. Consider a cochain complex $(C, d)$ in $K(\mathcal{C})$. For any graded lift $\bar{C}$ of $C^{\cdot}$, put

$$
\mathbf{C}=(\mathbf{C}, \bar{\delta})_{d, d}
$$

(1) There is an obstruction

$$
o\left(C^{\cdot}, d\right) \in H^{2} \mathbf{C}
$$

with

$$
o\left(C^{\cdot}, d\right)=0 \Longleftrightarrow L_{K(F)}\left(C^{\cdot}, d\right) \neq \varnothing .
$$

(2) If o $\left(C^{\cdot}, d\right)=0$, then $\operatorname{Sk}\left(L_{K(F)}\left(C^{\cdot}, d\right)\right)$ is affine over $H^{1} \mathbf{C}^{\text {. }}$.

Proof. This follows from combining Theorem 3.6 and Proposition 4.3 below.

Theorem 4.2. Consider a cochain map $f:\left(C^{*}, d_{C}\right) \longrightarrow\left(D^{*}, d_{D}\right)$ between cochain complexes in $\mathcal{C}$ and lifts $\left(\bar{C}, \bar{d}_{C}\right),\left(\bar{D}, \bar{d}_{D}\right)$ along $K(F)$. Put

$$
\mathbf{C}=(\mathbf{C}, \bar{\delta})_{F\left(\bar{d}_{C}\right), F\left(\bar{d}_{D}\right)}
$$


(1) There is an obstruction

$$
\begin{gathered}
o_{K(F)}\left(f \mid\left(\bar{C}, \bar{d}_{C}\right),\left(\bar{D}, \overline{d_{D}}\right)\right) \in H^{1} \mathbf{C} \\
\text { with } \\
o_{K(F)}\left(f \mid\left(\bar{C}, \bar{d}_{C}\right),\left(\bar{D}, \bar{d}_{D}\right)\right)=0 \Longleftrightarrow L_{K(F)}\left(f \mid\left(\bar{C}, \bar{d}_{C}\right),\left(\bar{D}, \bar{d}_{D}\right)\right) \neq \varnothing .
\end{gathered}
$$

(2) Suppose $H^{-1} \operatorname{Hom}\left(C^{\cdot}, D^{\cdot}\right)=0$. If $o_{K(F)}\left(f \mid\left(\bar{C}, \bar{d}_{C}\right),\left(\bar{D}, \bar{d}_{D}\right)\right)=0$, then $\operatorname{Sk}\left(L_{K(F)}\left(f \mid\left(\bar{C}, \bar{d}_{C}\right),\left(\bar{D}, \bar{d}_{D}\right)\right)\right)$ is affine over $H^{0} \mathbf{C}$.

Proof. This follows from combining Theorem 3.8 and Proposition 4.6 below.

Consider a cochain complex $\left(C^{\cdot}, d\right)$ in $K(\mathcal{C})$ and a graded lift $\bar{C}$ of $C$. Let $\tilde{L}_{F}(d \mid \bar{C})$ be the bigroupoid associated to the groupoid $L_{F}(d \mid \bar{C})$, i.e.

0 . Objects are differentials $\bar{d}$ on $\bar{C}$ making $(\bar{C}, \bar{d})$ into a lift of $(C \cdot d)$ along $C(F)$.

1. The morphisms from $\bar{d}$ to $\bar{d}^{\prime}$ are given by $\operatorname{Sk}\left(L_{F}\left(1: C^{\cdot} \longrightarrow C^{\cdot} \mid \bar{d}, \bar{d}^{\prime}\right)\right)$.

Proposition 4.3. The natural functor

$$
\Psi: \tilde{L}_{F}(d \mid \bar{C}) \longrightarrow L_{K(F)}(C, d): \bar{d} \longmapsto(\bar{C}, \bar{d})
$$

is essentially surjective and full. In particular, it induces a bijection

$$
\operatorname{Sk}\left(\tilde{L}_{F}(d \mid \bar{C})\right) \longrightarrow \operatorname{Sk}\left(L_{K(F)}\left(C^{\cdot}, d\right)\right) \text {. }
$$

If $H^{-1} \operatorname{Hom}\left(C^{\cdot}, D^{\cdot}\right)=0$, then $\Psi$ is an equivalence.

Proof. Let us prove essential surjectivity first. A lift of $\left(C^{\cdot}, d=d_{C}\right)$ along $K(F)$ consists of a homotopy equivalence $f:\left(C^{\cdot}, d_{C}\right) \longrightarrow\left(D^{*}, d_{D}\right)$ in $\mathcal{C}$ and a cochain complex $\left(\bar{D}, \bar{d}_{D}\right)$ in $\overline{\mathcal{C}}$ with $F(\bar{D})=D^{\prime}$ and $F\left(\bar{d}_{D}\right)=d_{D}$. By the crude lifting lemma of Corollary 3.11 there exist a $\bar{d}_{C} \in L_{F}\left(d \mid C^{\cdot}\right)$ and a homotopy equivalence $\bar{f} \in L_{F}\left(f \mid \bar{d}_{C}, \bar{d}_{D}\right)$. Consequently, $\left(\bar{D}, \bar{d}_{D}\right) \cong\left(\bar{C}, \bar{d}_{C}\right)$ in $L_{K(F)}\left(C^{\prime}, d\right)$. For the remainder of the proposition, we are to consider for $\bar{d}, \bar{d}^{\prime} \in L_{F}(d \mid \bar{C})$ the map

$$
\Phi: \operatorname{Sk}\left(L_{F}\left(1 \mid \bar{d}, \bar{d}^{\prime}\right)\right) \longrightarrow L_{K(F)}\left(1 \mid(\bar{C}, \bar{d}),\left(\bar{C}, \bar{d}^{\prime}\right)\right),
$$

hence the result follows from Proposition 4.6 below.

Corollary 4.4. Consider a cochain complex $\left(C^{\cdot}, d\right)$. The natural functor

$$
L_{C(F)}\left(C^{\cdot}, d\right) \longrightarrow L_{K(F)}\left(C^{\cdot}, d\right)
$$

is essentially surjective.

Corollary 4.5. The first part of Proposition 4.3 still holds if $\operatorname{Ker}(F)^{n}=0$.

Proof. This follows from Proposition 4.3 by induction.

Proposition 4.6. Consider a cochain map $f:\left(C^{\cdot}, d_{C}\right) \longrightarrow\left(D^{\cdot}, d_{D}\right)$ between cochain complexes in $\mathcal{C}$ and lifts $\left(\bar{C}, \bar{d}_{C}\right),\left(\bar{D}, \bar{d}_{D}\right)$ along $C(F)$. The natural map

$$
\Phi: \operatorname{Sk}\left(L_{F}\left(f \mid \bar{d}_{C}, \bar{d}_{D}\right)\right) \longrightarrow L_{K(F)}\left(f \mid\left(\bar{C}, \bar{d}_{C}\right),\left(\bar{D}, \bar{d}_{D}\right)\right)
$$

is surjective. If $H^{-1} \operatorname{Hom}\left(C^{\cdot}, D^{\cdot}\right)=0$, then it is a bijection.

Proof. An element in the image of $\Phi$ is given by a $\bar{g} \in L_{F}\left(g \mid \bar{d}_{C}, \bar{d}_{D}\right)$ for some $g$ homotopic to $f$. Suppose $H: f \longrightarrow g$ is a homotopy. By Proposition 3.12 (2), there exists some $\bar{f} \in L_{F}\left(f \mid \bar{d}_{C}, \bar{d}_{D}\right)$ for which there exists a homotopy $\bar{H}: \bar{f} \longrightarrow \bar{g}$, proving part one. For part two, suppose $\Phi(\bar{f})=\Phi\left(\bar{f}^{\prime}\right)$. So there exists a homotopy $\bar{H}: \bar{f} \longrightarrow \bar{f}^{\prime}$ lifting some homotopy $H: f \longrightarrow f$. Now $\bar{f}=\bar{f}^{\prime} \in \operatorname{Sk}\left(L_{F}\left(f \mid \bar{d}_{C}, \bar{d}_{D}\right)\right)$ if there exists a homotopy lifting $0: f \longrightarrow f$. By Proposition 3.12(1), this is the case if there exists a homotopy $\Lambda: 0 \longrightarrow H$, which finishes the proof. 


\section{Application to Linear AND ABElian DEFORMations}

In this section, we interpret the results of $\$ 3$ and 4 for linear and abelian deformations. Consider surjective ringmaps between coherent, commutative rings

$$
\bar{R} \longrightarrow R \longrightarrow R_{0}=S
$$

with $\operatorname{Ker}(\bar{R} \longrightarrow S)=I, \operatorname{Ker}(\bar{R} \longrightarrow R)=J$ and $I J=0$. In particular, $J^{2}=0$ and $J$ is an $S$-module.

5.1. Linear deformations. Let $\mathfrak{c}_{0}$ be a fixed flat $S$-linear category, i.e. its homsets are flat $S$-modules. A flat $R$-deformation of $\mathfrak{c}_{0}$ is an $R$-linear functor $\mathfrak{c} \longrightarrow \mathfrak{c}_{0}$ inducing an equivalence $S \otimes_{R} \mathfrak{c} \cong \mathfrak{c}_{0}$, where $\mathfrak{c}$ is flat over $R$. Here $S \otimes_{R} \mathfrak{c}$ is the $S$-linear category with the same objects as $\mathfrak{c}$ and $\left(S \otimes_{R} \mathfrak{c}\right)\left(C, C^{\prime}\right)=S \otimes_{R} \mathfrak{c}\left(C, C^{\prime}\right)$. Consider flat linear deformations

$$
\overline{\mathfrak{c}} \underset{F}{\longrightarrow} \mathfrak{c} \underset{(-)_{0}}{\longrightarrow} \mathfrak{c}_{0}
$$

along the given ringmaps. Since $J^{2}=0$, we have $\operatorname{Ker}(F)^{2}=0$ and $F$ is obviously essentially surjective and full.

Proposition 5.1. Consider pre-complexes $\left(C^{*}, d_{C}\right)$ and $\left(D^{*}, d_{D}\right)$ in $\mathfrak{c}$ with graded lifts $\bar{C}$ and $\bar{D}$ along $F$. The complex $\mathbf{C}=(\mathbf{C}, \bar{\delta})_{d_{C}, d_{D}}$ defined in (5) of 3.2 is

$$
\mathbf{C}=J \otimes_{S} \operatorname{Hom}_{\mathfrak{c}_{0}}\left(C_{0}^{\cdot}, D_{0}^{*}\right)
$$

where $\operatorname{Hom}_{\mathfrak{c}_{0}}\left(C_{0}^{\cdot}, D_{0}^{\cdot}\right)$ is endowed with the differential $\delta_{0}=\delta_{\left(d_{C}\right)_{0},\left(d_{D}\right)_{0}}$.

Proof. The complex $\left(\operatorname{Hom}_{\mathfrak{c}}\left(C^{\cdot}, D^{\cdot}\right), \delta\right)$ is by definition isomorphic to the complex $R \otimes \bar{R}_{R}\left(\operatorname{Hom}_{\overline{\mathfrak{c}}}(\bar{C}, \bar{D}), \bar{\delta}\right)$, so since $\overline{\mathfrak{c}}$ is a flat $\bar{R}$ linear category, the kernel in (5) is given by $J \otimes_{\bar{R}} \operatorname{Hom}_{\bar{c}}(\bar{C}, \bar{D})=J \otimes_{S} \operatorname{Hom}_{\mathfrak{c}_{0}}\left(C_{0}^{\cdot}, D_{0}^{*}\right)$ by change of rings.

Consequently, all the results of $\$ 3$ and $\$ 4$ can be reformulated using this complex. In particular, Theorems 4.1 and 4.2 yield the obstruction theory for lifting along $K(F)$ :

Theorem 5.2. Consider a cochain complex $C$ in $K(\mathfrak{c})$. Put

$$
\mathbf{C}=J \otimes_{S} \operatorname{Hom}_{\mathfrak{c}_{0}}\left(C_{0}^{\cdot}, C_{0}^{\cdot}\right) .
$$

(1) There is an obstruction

$$
o\left(C^{\cdot}\right) \in H^{2} \mathbf{C}
$$

with

$$
o\left(C^{\cdot}\right)=0 \Longleftrightarrow L_{K(F)}\left(C^{\cdot}\right) \neq \varnothing .
$$

(2) If $o\left(C^{\cdot}\right)=0$, then $\operatorname{Sk}\left(L_{K(F)}\left(C^{\cdot}\right)\right)$ is affine over $H^{1} \mathbf{C}$.

Theorem 5.3. Consider a cochain map $f: C \longrightarrow D$ between cochain complexes in $\mathfrak{c}$ and lifts $\bar{C}, \bar{D}$ along $K(F)$. Put

$$
\mathbf{C}=J \otimes_{S} \operatorname{Hom}_{\mathfrak{c}_{0}}\left(C_{0}^{\cdot}, D_{0}^{\cdot}\right) .
$$

(1) There is an obstruction

$$
o_{K(F)}(f \mid \bar{C}, \bar{D}) \in H^{1} \mathbf{C}
$$

with

$$
o_{K(F)}(f \mid \bar{C}, \bar{D})=0 \Longleftrightarrow L_{K(F)}(f \mid \bar{C}, \bar{D}) \neq \varnothing .
$$

(2) Suppose $H^{-1} \operatorname{Hom}_{\mathfrak{c}}\left(C^{\cdot}, D^{\cdot}\right)=0$. If $o_{K(F)}(f \mid \bar{C}, \bar{D})=0$, then

$$
\operatorname{Sk}\left(L_{K(F)}(f \mid \bar{C}, \bar{D})\right)
$$

is affine over $H^{0} \mathbf{C}$. 
5.2. Abelian deformations. We start with introducing some terminology for an $R$-linear abelian category $\mathcal{C}$. An object $C \in \mathcal{C}$ is called flat if the right-exact functor $-\otimes_{R} C: \bmod (R) \longrightarrow \mathcal{C}: R \longmapsto C$ is exact, and dually, $C$ is called coflat if the left-exact functor $\operatorname{Hom}_{R}(-, C): \bmod (R) \longrightarrow \mathcal{C}: R \longmapsto C$ is exact (mod denotes the finitely presented modules). The subcategories of flat and coflat objects are denoted by $\mathrm{FI}(\mathcal{C})$ and $\operatorname{Cof}(\mathcal{C})$ respectively. A (selfdual) notion of flatness for abelian categories was defined in [9, Def.3.2]. An abelian category with enough injectives is flat if its injectives are coflat. In general, a small abelian $\mathcal{C}$ is flat if its category of ind-objects, which is a category with enough injectives, is flat. This notion of flatness is different from the one used in $\$ 5.1$ for linear categories! However, we have the following connection:

(1) If $\mathfrak{c}$ is a small, flat $R$-linear category, then $\operatorname{Mod}(\mathfrak{c})=\operatorname{Add}(\mathfrak{c}, \operatorname{Ab})$ is flat as an abelian $R$-linear category.

(2) If $\mathcal{C}$ is a flat abelian $R$-linear category, then its category of injectives $\operatorname{lnj}(\mathcal{C})$ is flat as an $R$-linear category.

Now let $\mathcal{C}_{0}$ be a fixed flat abelian $S$-linear category. A flat abelian $R$-deformation of $\mathcal{C}_{0}$ is an $R$-linear functor $\mathcal{C}_{0} \longrightarrow \mathcal{C}$ inducing an equivalence $\mathcal{C}_{0} \cong \mathcal{C}_{S}$, where $\mathcal{C}$ is a flat abelian $R$-linear category. Here $\mathcal{C}_{S}$ denotes the category of $S$-objects in $\mathcal{C}$, i.e. objects $C$ with an $S$-structure $S \longrightarrow \mathcal{C}(C, C)$ extending the $R$-structure 9 . Def.5.2, $\S 4$. Consider flat abelian deformations along the given ringmaps, together with their adjoints:
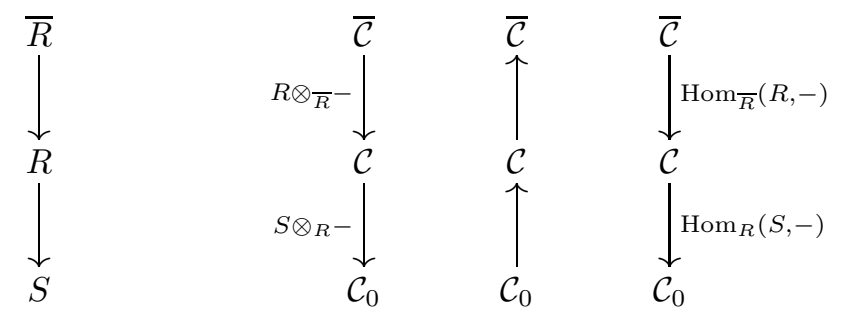

All our results for $\operatorname{Hom}_{\bar{R}}(R,-)$ have of course dual results for $R \otimes_{\bar{R}}-$.

Proposition 5.4. (1) $\operatorname{Ker}\left(\operatorname{Hom}_{\bar{R}}(R,-)\right)^{2}=0$.

(2) Consider pre-complexes $\left(C, d_{C}\right)$ and $\left(D^{*}, d_{D}\right)$ in $P(\operatorname{Cof}(\mathcal{C}))$ with graded lifts $\bar{C}$ and $\bar{D}$ in $G(\operatorname{Cof}(\overline{\mathcal{C}}))$ along $F=\operatorname{Hom}_{\bar{R}}(R,-)$. The complex $\mathbf{C}=$ $(\mathbf{C}, \bar{\delta})_{d_{C}, d_{D}}$ defined in (5) of 3.2 is

$$
\mathbf{C}^{\cdot}=\operatorname{Hom}_{\mathcal{C}_{0}}\left(\operatorname{Hom}_{R}\left(J, C^{\cdot}\right), \operatorname{Hom}_{R}\left(S, D^{*}\right)\right) .
$$

Proof. (1) For $\bar{C} \in \overline{\mathcal{C}}$, consider the exact sequence $0 \longrightarrow \operatorname{Hom}_{\bar{R}}(R, \bar{C}) \longrightarrow \bar{C} \longrightarrow$ $J \bar{C} \longrightarrow 0$ where $J \bar{C}$ is the image of $\bar{C} \longrightarrow \operatorname{Hom}_{\bar{R}}(J, \bar{C})$. A map $f: \bar{C} \longrightarrow \bar{D}$ has $\operatorname{Hom}_{\bar{R}}(R, f)=0$ if and only if $f$ factors as $\bar{C} \longrightarrow J \bar{C} \longrightarrow \operatorname{Hom}_{\bar{R}}(R, \bar{D}) \longrightarrow \bar{D}$. Clearly, any composition of two such maps is zero. (2) With the same argument, the kernel of (5) is given by $\operatorname{Hom}_{\mathcal{C}}\left(J \bar{C}, D^{*}\right)$, and under the flatness assumption on $\bar{C}, J \bar{C}=\operatorname{Hom}_{\bar{R}}(J, \bar{C})=\operatorname{Hom}_{R}\left(J, C^{\cdot}\right)$ by change of rings.

Consequently, restricting the codomain of $\operatorname{Hom}_{\bar{R}}(R,-)$ as in Remark 3.4 all the results of $\$ 3$ can be reformulated using this complex, In 6.4 we give the obstruction theory for lifting coflat objects and maps between them along $\operatorname{Hom}_{\bar{R}}(R,-)$, which yields of course an obstuction theory for lifting along $G\left(\operatorname{Hom}_{\bar{R}}(R,-)\right)$. Although we do not get a general obstruction theory for lifting along $K\left(\operatorname{Hom}_{\bar{R}}(R,-)\right): K(\overline{\mathcal{C}}) \longrightarrow$ $K(\mathcal{C})$ or along its restriction to coflat complexes, we $d o$ get an obstruction theory for its restriction to complexes of injectives if the category $\overline{\mathcal{C}}$, and hence also $\mathcal{C}$, 
has enough injectives. The reason is that in this case, as we will show in 5.3 Proposition [5.5] the functor

$$
\operatorname{Hom}_{\bar{R}}(R,-): \operatorname{lnj}(\overline{\mathcal{C}}) \longrightarrow \operatorname{lnj}(\mathcal{C})
$$

is a linear deformation, making lifting along $K\left(\operatorname{Hom}_{\bar{R}}(R,-)\right): K(\operatorname{Inj}(\overline{\mathcal{C}})) \longrightarrow$ $K(\operatorname{Inj}(\mathcal{C}))$ related to both $\$ 5.1$ and this section $\$ 5.2$

5.3. Lifting in the homotopy category of injectives. In this section we consider flat abelian deformations as in (8) of $\$ 5.2$ with enough injectives and we put

$$
F=\operatorname{Hom}_{\bar{R}}(R,-): \operatorname{Inj}(\overline{\mathcal{C}}) \longrightarrow \operatorname{Inj}(\mathcal{C}) .
$$

Proposition 5.5. Let $\mathcal{C}_{0} \longrightarrow \overline{\mathcal{C}}$ be a flat abelian deformation with enough injectives along $\bar{R} \longrightarrow S$. The functor $\operatorname{Hom}_{\bar{R}}(S,-): \operatorname{Inj}(\overline{\mathcal{C}}) \longrightarrow \operatorname{Inj}\left(\mathcal{C}_{0}\right)$ is a linear deformation.

Proof. For injective objects $\bar{E}, \bar{F}$ in $\overline{\mathcal{C}}$, it is easily seen that

$$
\operatorname{Hom}_{\overline{\mathcal{C}}}\left(\operatorname{Hom}_{\bar{R}}(X, \bar{E}), \bar{F}\right)=X \otimes_{\bar{R}} \operatorname{Hom}_{\overline{\mathcal{C}}}(\bar{E}, \bar{F})
$$

for any $X \in \bmod (\bar{R})$ since $\bar{E}$ is coflat by assumption on $\overline{\mathcal{C}}$. Applying this to $X=S$, we obtain

$$
\operatorname{Hom}_{\overline{\mathcal{C}}}\left(\operatorname{Hom}_{\bar{R}}(S, \bar{E}), \operatorname{Hom}_{\bar{R}}(S, \bar{F})\right)=S \otimes_{\bar{R}} \operatorname{Hom}_{\overline{\mathcal{C}}}(\bar{E}, \bar{F}) .
$$

So it remains to show that for every $\mathcal{C}_{0}$-injective object $E \in \mathcal{C}_{0}$ there exists an injective $\overline{\mathcal{C}}$-object $\bar{E}$ with $E \cong \operatorname{Hom}_{\bar{R}}(S, \bar{E})$. Let $E$ be an injective object of $\mathcal{C}_{0}$. Take a $\overline{\mathcal{C}}$-monomorphism $m: E \longrightarrow \overline{E^{\prime}}$ to a $\overline{\mathcal{C}}$-injective. We obtain a $\mathcal{C}_{0}$-monomorphism $s: E \longrightarrow E^{\prime}=\operatorname{Hom}_{\bar{R}}\left(S, \overline{E^{\prime}}\right)$. Since $E$ is injective in $\mathcal{C}_{S}$, we find $r: E^{\prime} \longrightarrow E$ with $r s=1_{E}$. This gives us an idempotent $e=s r: E^{\prime} \longrightarrow E^{\prime}$. Consider the map $\operatorname{Hom}_{\bar{R}}(S,-):\left(\overline{E^{\prime}}, \overline{E^{\prime}}\right) \longrightarrow\left(E^{\prime}, E^{\prime}\right)$. By (10), this map has a nilpotent kernel $I\left(\overline{E^{\prime}}, \overline{E^{\prime}}\right)$. It follows that the idempotent $e$ lifts to an idempotent $\bar{e}$ in $\left(\overline{E^{\prime}}, \overline{E^{\prime}}\right)$. This idempotent $\bar{e}$ splits as $\bar{e}=\bar{s} \bar{r}$ with $\bar{r}: \overline{E^{\prime}} \longrightarrow \bar{E}, \bar{s}: \bar{E} \longrightarrow \overline{E^{\prime}}$ for a $\mathcal{C}$-injective $\bar{E}$. We now obviously find an isomorphism $E \cong \operatorname{Hom}_{\bar{R}}(S, \bar{E})$.

In accordance with (92), both Propositions [5.1] and 5.4 now yield

Proposition 5.6. Consider pre-complexes of injectives $\left(C^{\cdot}, d_{C}\right)$ and $\left(D^{\cdot}, d_{D}\right)$ in $\mathcal{C}$ with graded lifts of injectives $\bar{C}$ and $\bar{D}$ along $\operatorname{Hom}_{\bar{R}}(R,-)$. The complex $\mathbf{C}=$ $(\mathbf{C}, \bar{\delta})_{d_{C}, d_{D}}$ defined in (5) of 3.2 is

$$
\begin{aligned}
\mathbf{C} & =J \otimes_{S} \operatorname{Hom}_{\mathcal{C}_{0}}\left(\operatorname{Hom}_{R}\left(S, C^{\cdot}\right), \operatorname{Hom}_{R}\left(S, D^{\cdot}\right)\right) \\
& =\operatorname{Hom}_{\mathcal{C}_{0}}\left(\operatorname{Hom}_{R}\left(J, C^{\cdot}\right), \operatorname{Hom}_{R}\left(S, D^{*}\right)\right) .
\end{aligned}
$$

Theorems 5.2 and 5.3 yield:

Theorem 5.7. Consider a cochain complex $C$ in $K(\operatorname{Inj}(\mathcal{C}))$. Put

$$
\mathbf{C}=\operatorname{Hom}_{\mathcal{C}_{0}}\left(\operatorname{Hom}_{R}\left(J, C^{\cdot}\right), \operatorname{Hom}_{R}\left(S, C^{\cdot}\right)\right) .
$$

(1) There is an obstruction

$$
o\left(C^{\cdot}\right) \in H^{2} \mathbf{C}
$$

with

$$
o\left(C^{\cdot}\right)=0 \Longleftrightarrow L_{K(F)}\left(C^{\cdot}\right) \neq \varnothing .
$$

(2) If $o\left(C^{\cdot}\right)=0$, then $\operatorname{Sk}\left(L_{K(F)}\left(C^{\cdot}\right)\right)$ is affine over $H^{1} \mathbf{C}^{\text {. }}$.

Theorem 5.8. Consider a cochain map $f: C \longrightarrow D$ between cochain complexes in $\operatorname{lnj}(\mathcal{C})$ and lifts $\bar{C}, \bar{D}$ along $K(F)$. Put

$$
\mathbf{C}=\operatorname{Hom}_{\mathcal{C}_{0}}\left(\operatorname{Hom}_{R}\left(J, C^{\cdot}\right), \operatorname{Hom}_{R}\left(S, D^{\cdot}\right)\right) .
$$


(1) There is an obstruction

$$
o_{K(F)}(f \mid \bar{C}, \bar{D}) \in H^{1} \mathbf{C}
$$

with

$$
o_{K(F)}(f \mid \bar{C}, \bar{D})=0 \Longleftrightarrow L_{K(F)}(f \mid \bar{C}, \bar{D}) \neq \varnothing .
$$

(2) Suppose $H^{-1} \operatorname{Hom}_{\mathcal{C}}\left(C^{\cdot}, D^{\cdot}\right)=0$. If $o_{K(F)}(f \mid \bar{C}, \bar{D})=0$, then

$$
\operatorname{Sk}\left(L_{K(F)}(f \mid \bar{C}, \bar{D})\right)
$$

is affine over $H^{0} \mathbf{C}$.

\section{DeRIVED LifTing}

In this section, we use the results of section $\$ 5.3$ to obtain obstruction theories for derived lifting along the adjoints of an abelian deformation (Theorems 6.3 6.4 and Theorem 6.10). This eventually leads to the obstruction theory for coflat objects (Theorems 6.11, 6.12).

6.1. Comparing lift groupoids. In the sequel, we will often compare lift groupoids as in Definition 3.1 of lifts along different functors. We will use the following technical tool:

Definition 6.1. We will say that a diagram of functors

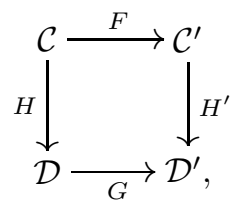

satisfies (L) if the following conditions are fulfilled:

(1) The diagram is commutative up to natural isomorphism.

(2) $F$ and $G$ are fully faithful.

(3) If $H^{\prime}\left(C^{\prime}\right) \cong G(D)$, then there is a $C \in \mathcal{C}$ with $C^{\prime} \cong F(C)$.

Proposition 6.2. Suppose a diagram as in Definition 6.1 satisfies $(L)$.

(1) For $D \in \mathcal{D}$ and $D^{\prime} \cong G(D)$ in $\mathcal{D}^{\prime}$, there is an equivalence of groupoids $L_{H}(D) \longrightarrow L_{H^{\prime}}\left(D^{\prime}\right)$.

(2) For $f: D_{1} \longrightarrow D_{2}$ in $\mathcal{D}, g \cong G(f)$ in $\mathcal{D}^{\prime}, C_{1} \in L_{H}\left(D_{1}\right), C_{1}^{\prime} \cong F\left(C_{1}\right)$, $C_{2} \in L_{H}\left(D_{2}\right)$ and $C_{2}^{\prime} \cong F\left(C_{2}\right)$ and $g \cong G(f)$ in $\mathcal{D}^{\prime}$, there is a bijection $L_{H}\left(f \mid C_{1}, C_{2}\right) \longrightarrow L_{H^{\prime}}\left(g \mid C_{1}^{\prime}, C_{2}^{\prime}\right)$.

Proof. The proofs of 1 and 2 are similar and easy. For example for 1, it is convenient to consider the category $\mathcal{L}$ with as objects functors $H$ with a specified object in the codomain of $H$ and maps between $(H: \mathcal{C} \longrightarrow \mathcal{D}, D)$ and $\left(H^{\prime}: \mathcal{C}^{\prime} \longrightarrow \mathcal{D}^{\prime}, D^{\prime}\right)$ given by 4-tupels $(F, G, \eta, f)$ in which $F$ and $G$ fit into a square as in Definition 6.1 (but not necessarily satisfying $(\mathrm{L})), \eta$ is a natural isomorphism $\eta: G H \cong H^{\prime} F$, and $f$ is an isomorphism $f: D^{\prime} \cong G(D)$. There is seen to be a lift functor $L: \mathcal{L} \longrightarrow \mathrm{Gd}$ mapping $(H, D)$ to $L_{H}(D)$. If the square satisfies $(\mathrm{L}), L(F, G, \eta, f)$ is easily seen to be an equivalence. 
6.2. Derived lifting with enough injectives. In this section we consider flat abelian deformations as in (8) of $\$ 5.2$ with enough injectives and we consider the derived functor

$$
F=R \operatorname{Hom}_{\bar{R}}(R,-): D^{+}(\overline{\mathcal{C}}) \longrightarrow D^{+}(\mathcal{C}) .
$$

We can now easily deduce the obstruction theory for $F$ from Theorems 5.7 and 5.8

Theorem 6.3. Consider a cochain complex $C^{\cdot} \in D^{+}(\mathcal{C})$. Put

$$
\mathbf{C}=R \operatorname{Hom}_{\mathcal{C}_{0}}\left(R \operatorname{Hom}_{R}\left(J, C^{\cdot}\right), R \operatorname{Hom}_{R}\left(S, C^{\cdot}\right)\right) .
$$

(1) There is an obstruction

$$
o\left(C^{\cdot}\right) \in H^{2} \mathbf{C}
$$

with

$$
o\left(C^{\cdot}\right)=0 \Longleftrightarrow L_{F}\left(C^{\cdot}\right) \neq \varnothing .
$$

(2) If o $\left(C^{\cdot}\right)=0$, then $\operatorname{Sk}\left(L_{F}\left(C^{\cdot}\right)\right)$ is affine over $H^{1} \mathbf{C}^{\cdot}$.

Theorem 6.4. Consider a cochain map $f: C \cdot D$ between cochain complexes in $D^{+}(\mathcal{C})$ and lifts $\bar{C}, \bar{D}$ along $F$. Put

$$
\mathbf{C}^{\cdot}=R \operatorname{Hom}_{\mathcal{C}_{0}}\left(R \operatorname{Hom}_{R}\left(J, C^{\cdot}\right), R \operatorname{Hom}_{R}\left(S, D^{*}\right)\right) .
$$

(1) There is an obstruction

$$
o(f \mid \bar{C}, \bar{D}) \in H^{1} \mathbf{C}
$$

with

$$
o(f \mid \bar{C}, \bar{D})=0 \Longleftrightarrow L_{F}(f \mid \bar{C}, \bar{D}) \neq \varnothing .
$$

(2) Suppose $\operatorname{Ext}_{\mathcal{C}}^{-1}\left(C^{\cdot}, D^{\cdot}\right)=0$. If $o(f \mid \bar{C}, \bar{D})=0$, then $\operatorname{Sk}\left(L_{F}(f \mid \bar{C}, \bar{D})\right)$ is affine over $H^{0} \mathbf{C}$.

We use the following:

Proposition 6.5. In the diagram

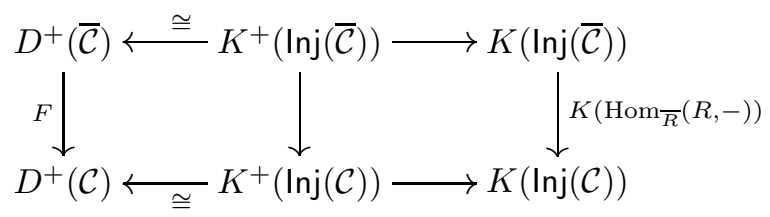

both squares satisfy $(L)$. Consequently, for $C \in D^{+}(\mathcal{C})$, there is an isomorphism $C \cdot \cong E \cdot$ with $E^{\cdot} \in K^{+}(\operatorname{Inj}(\mathcal{C}))$ and an equivalence

$$
L_{K\left(\operatorname{Hom}_{R}(S,-)\right)}\left(E^{\cdot}\right) \sim L_{F}\left(C^{\cdot}\right)
$$

and likewise for maps (see Proposition 6.2).

Proof. Obvious.

Proof of Theorems 6.3 and 6.4 Consider $C^{\cdot}, D^{\cdot} \in D^{+}(\mathcal{C})$ and isomorphisms $C^{\cdot} \cong$ $E^{\cdot}$ and $D^{\cdot} \cong F^{\cdot}$ with $E^{\cdot}, F^{\cdot} \in K^{+}(\operatorname{Inj}(\mathcal{C}))$. We have

$\operatorname{Hom}_{\mathcal{C}_{0}}\left(\operatorname{Hom}_{R}\left(J, E^{\cdot}\right), \operatorname{Hom}_{R^{\prime}}\left(S, F^{\cdot}\right)\right)=R \operatorname{Hom}_{\mathcal{C}_{0}}\left(R \operatorname{Hom}_{R}\left(J, C^{\cdot}\right), R \operatorname{Hom}_{R^{\prime}}\left(S, D^{\cdot}\right)\right)$, hence the proof follows by combining Theorems 5.7 and 5.8 and Proposition 6.5 
Remark 6.6. If $\overline{\mathcal{C}}$ is a Grothendieck category, one could hope to deduce an obstruction theory for the unbounded derived categories $D(\overline{\mathcal{C}}) \longrightarrow D(\mathcal{C})$ from the restriction of $K\left(\operatorname{Hom}_{\bar{R}}(R,-)\right)$ to homotopically injective complexes of injectives. Unfortunately not every lift of a homotopically injective complex is homotopically injective. The canonical counter example is given by $\bar{R}=\mathbb{Z} / p^{2} \mathbb{Z}, R=\mathbb{Z} / p \mathbb{Z}$ and the $R$-complex

$$
\cdots \rightarrow \mathbb{Z} / p \mathbb{Z} \stackrel{0}{\rightarrow} \mathbb{Z} / p \mathbb{Z} \stackrel{0}{\rightarrow} \mathbb{Z} / p \mathbb{Z} \rightarrow \cdots
$$

which lifts to the non-homotopically injective complex

$$
\cdots \rightarrow \mathbb{Z} / p^{2} \mathbb{Z} \stackrel{p}{\rightarrow} \mathbb{Z} / p^{2} \mathbb{Z} \stackrel{p}{\rightarrow} \mathbb{Z} / p^{2} \mathbb{Z} \rightarrow \cdots
$$

In general it is unclear to us if a homotopically injective complex in $K(\operatorname{lnj}(\mathcal{C}))$ always has a homotopically injective lift to $K(\operatorname{lnj}(\overline{\mathcal{C}}))$. In the bounded below case, this problem is overcome by the fact that being a complex of injectives is a property on the graded level.

6.3. Lifting complexes of bounded coflat dimension. Consider flat abelian deformations as in (8) of \$5.2 of small abelian categories, and consider the associated deformations of ind-objects, which have enough injectives. We will discuss some restrictions of the derived functor

$$
R \operatorname{Hom}_{\bar{R}}(R,-): D^{+}(\operatorname{Ind} \overline{\mathcal{C}}) \longrightarrow D^{+}(\operatorname{Ind} \mathcal{C})
$$

of the previous section, for which (the restrictions of) Theorems 6.3 and 6.4 still hold (Theorem 6.10). Since by enlarging the universe, we may assume that any category is small, the results of this section hold for arbitrary abelian categories.

In general, $R \operatorname{Hom}_{R}(-,-)$ and $\operatorname{Ext}_{R}^{i}(-,-)$ are defined as derived functors in the first argument, fixing the second one. However we will use the following double interpretation in the sequel:

Proposition 6.7. If $\mathcal{C}$ is a flat $R$-linear abelian category with enough injectives, then we have a derived bifunctor

$$
D^{-}(\bmod (R)) \times D^{+}(\mathcal{C}) \longrightarrow D^{+}(\mathcal{C}):\left(M ., C^{\cdot}\right) \longmapsto R \operatorname{Hom}_{R}\left(M ., C^{\cdot}\right) .
$$

If $P . \longrightarrow M$. is a bounded above projective resolution of $M$. in $\bmod (R)$, and $C . \longrightarrow$ $E^{\cdot}$ is a bounded below injective resolution of $C$ in $\mathcal{C}$, we have

$$
R \operatorname{Hom}_{R}\left(M_{.}, C^{\cdot}\right)=\operatorname{Hom}_{R}\left(P ., C^{\cdot}\right) \cong \operatorname{Hom}_{R}\left(P ., E^{\cdot}\right) \cong \operatorname{Hom}_{R}\left(M ., E^{\cdot}\right) .
$$

Proof. This is just the classical proof, since $\mathcal{C}$-injectives are coflat and for projectives $P$ in $\bmod (R), \operatorname{Hom}_{R}(P,-)$ is exact.

Definition 6.8. For a complex $C \in C(\mathcal{C})$, its coflat dimension is defined to be

$$
\operatorname{cd}\left(C^{*}\right)=\min \left\{n \in \mathbb{N}|\forall M \in \bmod (R), \forall| i \mid>n \operatorname{Ext}_{R}^{i}\left(M, C^{*}\right)=0\right\}
$$

if such an $n$ exists and $\operatorname{cd}\left(C^{\cdot}\right)=\infty$ otherwise.

Note that since $\operatorname{Ext}_{R}^{i}(M,-)$ in $\mathcal{C}$ and $\operatorname{Ind} \mathcal{C}$ coincide, $\operatorname{cd}_{\mathcal{C}}\left(C^{\cdot}\right)=\operatorname{cd}_{\operatorname{lnd} \mathcal{C}}\left(C^{\cdot}\right)$. We consider the following full subcategories of $D^{+}(\mathcal{C})$ and $D^{+}(\operatorname{Ind} \mathcal{C})$ respectively:

- $\left|D_{\text {cd } \leq n}^{+}(\mathcal{C})\right|=\left\{C^{\cdot} \in D^{+}(\mathcal{C}) \mid \operatorname{cd}\left(C^{\cdot}\right) \leq n\right\} \quad(n \in \mathbb{N})$

- $\left|D_{\text {fcd }}^{+}(\mathcal{C})\right|=\left\{C^{\cdot} \in D^{+}(\mathcal{C}) \mid \operatorname{cd}\left(C^{\cdot}\right)<\infty\right\}$

- $\left|D_{\mathcal{C}, \mathrm{cd} \leq n}^{+}(\operatorname{lnd} \mathcal{C})\right|=\left\{C^{\cdot} \in D_{\mathcal{C}}^{+}(\operatorname{lnd} \mathcal{C}) \mid \operatorname{cd}\left(C^{\cdot}\right) \leq n\right\} \quad(n \in \mathbb{N})$

- $\left|D_{\mathcal{C}, \text { fcd }}^{+}(\operatorname{Ind} \mathcal{C})\right|=\left\{C^{\cdot} \in D_{\mathcal{C}}^{+}(\operatorname{Ind} \mathcal{C}) \mid \operatorname{cd}\left(C^{\cdot}\right)<\infty\right\}$ 
Proposition 6.9. There is a diagram

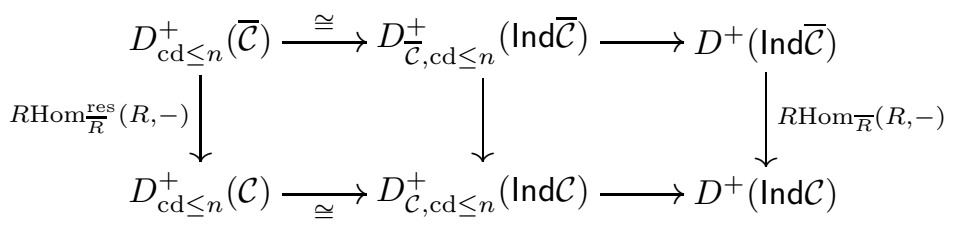

in which both squares satisfy $(L)$, and a similar diagram with "cd $\leq n$ " replaced by "fcd" with the same property. Consequently, in both cases, for $C^{\cdot} \in D_{\mathrm{cd} \leq n}^{+}(\mathcal{C})$, there is an equivalence

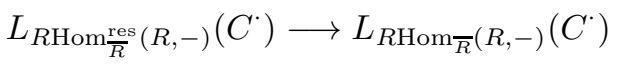

and likewise for maps (see Proposition 6.2).

Proof. For $C^{\cdot} \in D_{\overline{\mathcal{C}}, \mathrm{cd} \leq n}^{+}(\operatorname{Ind} \overline{\mathcal{C}})$, we have $\operatorname{cd}_{\mathcal{C}}\left(R \operatorname{Hom}_{\bar{R}}\left(R, C^{\cdot}\right)\right) \leq n$ by change of rings for $R$ Hom. Also, by the equivalence $D^{b}(\overline{\mathcal{C}}) \longrightarrow D_{\overline{\mathcal{C}}}^{b}(\operatorname{Ind} \overline{\mathcal{C}})$, $\operatorname{Ext} \frac{i}{R}\left(R, C^{\cdot}\right)$ is in $\mathcal{C}$. This yields the middle vertical arrow. The arrow $R \operatorname{Hom} \frac{\text { res }}{R}(R,-)$ is obtained using the two horizontal equivalences. Next, we show that the right diagram satisfies (L). There are two points to be checked. Suppose $C^{\cdot} \in D^{+}(\operatorname{Ind} \overline{\mathcal{C}})$ is such that $R \operatorname{Hom}_{\bar{R}}\left(R, C^{\cdot}\right)$ is in $D_{\mathcal{C}, \mathrm{cd} \leq n}^{+}(\operatorname{Ind} \mathcal{C})$. First, to show that $H^{i} C^{\cdot} \in \overline{\mathcal{C}}$, we use that Ind $\overline{\mathcal{C}}$ is a locally coherent $\mathrm{Ab} 5$ category with $\overline{\mathcal{C}}$ as finitely presented objects. We use the long exact cohomology sequence $\ldots \longrightarrow \operatorname{Ext}_{\bar{R}}^{i-1}\left(J, C^{\cdot}\right) \longrightarrow \operatorname{Ext}_{\bar{R}}\left(R, C^{\cdot}\right) \longrightarrow$ $H^{i} C \longrightarrow \operatorname{Ext}_{\frac{i}{R}}\left(J, C^{\cdot}\right) \longrightarrow \operatorname{Ext}_{\frac{i+1}{R}}\left(R, C^{\cdot}\right) \longrightarrow \ldots$ By the equivalence $D^{b}(\mathcal{C}) \longrightarrow$ $D_{\mathcal{C}}^{b}(\operatorname{Ind} \mathcal{C}), \operatorname{Ext}_{\bar{R}}^{i}\left(J, C^{\cdot}\right)=\operatorname{Ext}_{S}^{i}\left(J, R \operatorname{Hom}_{R}\left(S, C^{\cdot}\right)\right)$ is finitely presented, hence so is $H^{i} C$ as an extension of finitely presented objects. Next, we need to show that $\operatorname{cd}\left(C^{\cdot}\right) \leq n$. Writing an arbitrary $M \in \bmod (\bar{R})$ as an extension of modules in $\bmod (R)$, it follows from the associated long exact Ext sequence that it suffices to prove $\operatorname{Ext}_{\bar{R}}\left(M, C^{\cdot}\right)=0$ for $|i|>n$ and $M \in \bmod (R)$. But this follows from the assumption on $R \operatorname{Hom}_{\bar{R}}\left(R, C^{\cdot}\right)$ since $\operatorname{Ext} \frac{i}{\bar{R}}\left(M, C^{\cdot}\right)=\operatorname{Ext}_{R}^{i}\left(M, R \operatorname{Hom}_{\bar{R}}\left(R, C^{\cdot}\right)\right)$.

Theorem 6.10. Consider $C^{\cdot} \in D_{\mathrm{cd} \leq n}^{+}(\mathcal{C})$. Theorems 6.3 and 6.4 hold for

$$
F=R \operatorname{Hom} \frac{\mathrm{res}}{R}(R,-): D_{\mathrm{cd} \leq n}^{+}(\overline{\mathcal{C}}) \longrightarrow D_{\mathrm{cd} \leq n}^{+}(\mathcal{C})
$$

and for

$$
F=R \operatorname{Hom} \frac{\text { res }}{R}(R,-): D_{\text {fcd }}^{+}(\overline{\mathcal{C}}) \longrightarrow D_{\text {fcd }}^{+}(\mathcal{C})
$$

Proof. This follows from Theorems 6.3 and 6.4 and Proposition 6.9

6.4. Lifting coflat objects. In this section we consider arbitrary flat abelian deformations as in (8) of $\$ 5.2$ and we consider

$$
F=\operatorname{Hom}_{\bar{R}}(R,-): \operatorname{Cof}(\overline{\mathcal{C}}) \longrightarrow \operatorname{Cof}(\mathcal{C}) .
$$

Theorem 6.11. Consider $C \in \operatorname{Cof}(\mathcal{C})$. Put

$$
\mathbf{C}=R \operatorname{Hom}_{\mathcal{C}_{0}}\left(R \operatorname{Hom}_{R}(J, C), R \operatorname{Hom}_{R}(S, C)\right) .
$$

(1) There is an obstruction

$$
o(C) \in H^{2} \mathbf{C}
$$

with

$$
o(C)=0 \Longleftrightarrow L_{F}(C) \neq \varnothing .
$$

(2) If $o(C)=0$, then $\operatorname{Sk}\left(L_{F}(C)\right)$ is affine over $H^{1} \mathbf{C}$. 
Theorem 6.12. Consider a map $f: C \longrightarrow D$ in $\operatorname{Cof}(\mathcal{C})$ and lifts $\bar{C}, \bar{D}$ along $F$. Put

$$
\mathbf{C}^{\cdot}=R \operatorname{Hom}_{\mathcal{C}_{0}}\left(R \operatorname{Hom}_{R}(J, C), R \operatorname{Hom}_{R}(S, D)\right) .
$$

(1) There is an obstruction

$$
o(f \mid \bar{C}, \bar{D}) \in H^{1} \mathbf{C}
$$

with

$$
o(f \mid \bar{C}, \bar{D})=0 \Longleftrightarrow L_{F}(f \mid \bar{C}, \bar{D}) \neq \varnothing .
$$

(2) If o $(f \mid \bar{C}, \bar{D})=0$, then $\operatorname{Sk}\left(L_{F}(f \mid \bar{C}, \bar{D})\right)$ is affine over $H^{0} \mathbf{C}$.

Proposition 6.13. The diagram

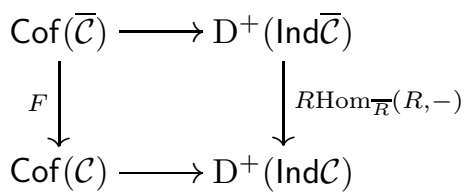

satisfies (L). Consequently, for $C \in \operatorname{Cof}(\mathcal{C})$, there is an equivalence

$$
L_{F}(C) \longrightarrow L_{R \mathrm{Hom}_{\bar{R}}^{(R,-)}}(C)
$$

and likewise for maps.

Proof. By Proposition 6.9 for $n=0$, it suffices to note that there is a diagram

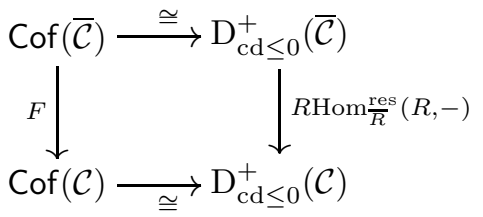

which obviously satisfies (L).

Proof of Theorems 6.11 and 6.12. This follows by combining Theorems 6.3 and 6.4 and Proposition 6.13

\section{Appendix: Miniversal Deformations}

In this appendix we prove the existence of miniversal deformations in the classical setting of [12] using [12, Theorem 2.11]. The results in 77.187 .2 are well known [8]. The results in $\$ 7.3$ can be found in [7 for the derived category of coherent sheaves over a projective variety and in 2 for the derived category of a profinite group.

Let $S=R_{0}=k$ be a field, let $\hat{\mathbf{C}}$ be the category of complete noetherian local $k$-algebras $(A, \mathrm{~m})$ with residue field $k$ and let $\mathbf{C}$ be its subcategory of artinian rings. Let $F: \mathbf{C} \longrightarrow$ Set be a functor such that $F(k)$ is a singleton. Recall that a hull for $F$ [12, Def 2.7] is a natural transformation $\eta: H=\hat{\mathbf{C}}(R,-) \longrightarrow F$ (for some $R \in \hat{\mathbf{C}}$ ) such that

(H1) $\eta$ is formally smooth [12, Def 2.2]; i.e. every surjective $\mathbf{C}$-map $R^{\prime} \longrightarrow R$ induces a surjection $H\left(R^{\prime}\right) \longrightarrow H(R) \times_{F(R)} F\left(R^{\prime}\right)$.

(H2) $\eta_{k[\epsilon] /\left(\epsilon^{2}\right)}: H\left(k[\epsilon] /\left(\epsilon^{2}\right)\right) \longrightarrow F\left(k[\epsilon] / \epsilon^{2}\right)$ is a bijection.

If we extend $F$ to $\hat{\mathbf{C}}$ by putting $\hat{F}((A, \mathrm{~m}))=\operatorname{projlim} F\left(A / \mathrm{m}^{n}\right)$, a hull for $F$ corresponds to an element $\zeta=\eta(1) \in \hat{F}(R)$, which is called a miniversal deformation of the unique element of $F(k)$. If $\eta$ is a natural isomorphism, $F$ is called prorepresentable, and in this case $\zeta$ is a universal deformation. Schlessingers conditions [12. Theorem 2.11] for the existence of a hull are 
(S1) If $R^{\prime} \longrightarrow R$ is a surjective $\mathbf{C}$-map with kernel of dimension 1, and if $R^{\prime \prime} \longrightarrow$ $R$ is any $\mathbf{C}$-map, then the map

$$
F\left(R^{\prime} \times_{R} R^{\prime \prime}\right) \longrightarrow F\left(R^{\prime}\right) \times_{F(R)} F\left(R^{\prime \prime}\right)
$$

is surjective.

(S2) The map (11) is bijective when $R^{\prime} \longrightarrow R$ is $k[\epsilon] / \epsilon^{2} \longrightarrow k$.

(S3) The tangent space $F\left(k[\epsilon] /\left(\epsilon^{2}\right)\right)$ is a finite dimensional $k$-vector space.

If in addition the maps in (S1) are bijective, then $F$ is pro-representable.

7.1. Deformations of differentials. Let $\mathfrak{c}$ be a fixed $k$-linear category. For $R \in$ $\mathbf{C}$, we consider the trivial $R$-deformation $F_{R}: R \otimes_{k} \mathfrak{c} \longrightarrow \mathfrak{c}$ of $\mathfrak{c}$. For a fixed complex $C^{\cdot}=\left(C^{\cdot}, d\right) \in C(\mathfrak{c})$, we consider $C_{R}=C^{\cdot}$ as fixed graded lift to $R \otimes_{k} \mathfrak{c}$. Put $L(R)=L_{F_{R}}\left(d \mid C^{\cdot}\right)$ as in Definition [3.2(3). Consequently, $|L(R)|$ contains all lifts of $d$ to a differential $d_{R}$ on $C$ in $R \otimes_{k} \mathfrak{c}$, and in $\operatorname{Sk}(L(R))$ two such lifts $d_{R}$ and $d_{R}^{\prime}$ are equivalent if there exists a lift of $1: d \longrightarrow d$ to an isomorphism $d_{R} \longrightarrow d_{R}^{\prime}$. We will call the objects of $L(R)(R$-)deformations of $d$. We consider the functor

$$
F: \mathbf{C} \longrightarrow \operatorname{Set}: R \longmapsto \operatorname{Sk}(L(R)) \text {. }
$$

Proposition 7.1. If $\operatorname{dim}_{k}\left(K(\mathfrak{c})\left(C^{\cdot}, C^{\cdot}[1]\right)\right)<\infty$, then $F$ has a hull; in other words, the differential $d$ has a miniversal deformation.

Proof. Consider the commutative diagram

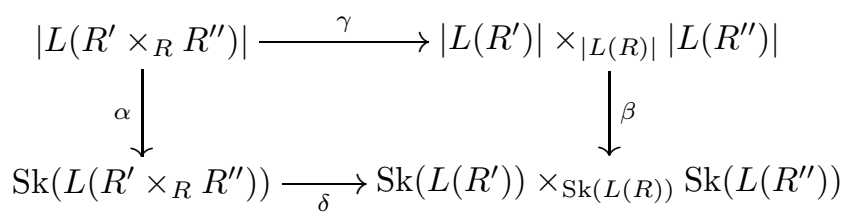

By Lemma 7.2(1), $\gamma$ is a bijection and it easily follows from Lemma 7.2 (2) that $\beta$ is surjective if $R^{\prime} \longrightarrow R$ is surjective with kernel of dimension 1. Consequently, (H1) holds for $F$. For $R=k$, both $|L(R)|$ and $\operatorname{Sk}(L(R))$ are singletons, which easily implies that $\delta$ is a bijection hence $(\mathrm{H} 2)$ holds for $F$. Finally, $(H 3)$ follows from Proposition 5.1] Theorem 3.8 and the assumption.

We have used the following Lemma.

Lemma 7.2. Consider the functor

$$
F_{0}: \mathbf{C} \longrightarrow \text { Set }: R \longmapsto|L(R)|
$$

and the canonical natural transformation

$$
\mu: F_{0} \longrightarrow F \text {. }
$$

(1) For arbitrary $\mathbf{C}$-maps $R^{\prime} \longrightarrow R$ and $R^{\prime \prime} \longrightarrow R$, the map

$$
F_{0}\left(R^{\prime} \times_{R} R^{\prime \prime}\right) \longrightarrow F_{0}\left(R^{\prime}\right) \times_{F_{0}(R)} F_{0}\left(R^{\prime \prime}\right)
$$

of (S1) is bijective.

(2) $\mu$ is formally smooth.

Proof. (1) By flatness of $\mathfrak{c}(C, D)$ over $k$, the canonical

$$
\left(R^{\prime} \times_{R} R^{\prime \prime}\right) \otimes_{k} \mathfrak{c}(C, D) \longrightarrow R^{\prime} \otimes_{k} \mathfrak{c}(C, D) \times_{R \otimes_{k} \mathfrak{c}(C, D)} R^{\prime \prime} \otimes_{k} \mathfrak{c}(C, D)
$$

is an isomorphism of $k$-modules. Endowing the right hand side with componentwise compositions, there results an isomorphism of categories

$$
\left(R^{\prime} \times_{R} R^{\prime \prime}\right) \otimes_{k} \mathfrak{c} \longrightarrow R^{\prime} \otimes_{k} \mathfrak{c} \times_{R \otimes_{k} \mathfrak{c}} R^{\prime \prime} \otimes_{k} \mathfrak{c} .
$$

Consequently, under (12), a graded lift $d^{\prime \prime \prime}$ of $d$ to $\left(R^{\prime} \times_{R} R^{\prime \prime}\right) \otimes_{k} \mathfrak{c}$ corresponds to a couple $\left(d^{\prime}, d^{\prime \prime}\right)$ of graded lifts $d^{\prime}$ of $d$ to $R^{\prime} \otimes_{k} \mathfrak{c}$ and $d^{\prime \prime}$ of $d$ to $R^{\prime \prime} \otimes_{k} \mathfrak{c}$ with the 
same image in $R \otimes_{k}$ c. Furthermore, $d^{\prime \prime \prime}{ }^{2}$ corresponds to $\left(d^{\prime 2}, d^{\prime \prime 2}\right)$, which finishes the proof of (1). (2) We are to show that a surjection $R \longrightarrow S$ in $\mathbf{C}$ induces a surjection $F(R) \longrightarrow F(S) \otimes_{F_{0}(S)} F_{0}(R)$. So suppose we have lifted differentials $d_{S}$ on $C_{S}^{\cdot}, d_{R}$ on $C_{R}^{*}$ with an isomorphism $f:\left.d_{S} \longrightarrow\left(d_{R}\right)\right|_{S}$ lifting 1 . Then there exists a differential $d_{R}^{\prime}$ lifting $d_{S}$ and an isomorphism $d_{R}^{\prime} \longrightarrow d_{R}$ lifting $f$ (this follows by induction on the kernel of $R \longrightarrow S$ from Corollary 3.11 and Proposition 3.3).

7.2. Homotopy deformations. Let $\mathfrak{c}$ and $F_{R}: R \otimes_{k} \mathfrak{c} \longrightarrow \mathfrak{c}$ be as in the previous section and consider $K\left(F_{R}\right): K\left(R \otimes_{k} \mathfrak{c}\right) \longrightarrow K(\mathfrak{c})$. For $C \in K(\mathfrak{c})$, we will call the objects of $L_{K\left(F_{R}\right)}\left(C^{\cdot}\right)$ homotopy $\left(R\right.$-) deformations of $C^{\cdot}$. We consider the functor

$$
F_{1}: \mathbf{C} \longrightarrow \operatorname{Set}: R \longmapsto \operatorname{Sk}\left(L_{K\left(F_{R}\right)}\left(C^{\cdot}\right)\right) \text {. }
$$

Proposition 7.3. There is a natural isomorphism of functors $F_{0} \cong F_{1}$. Consequently, if $\operatorname{dim}_{k}\left(K(\mathfrak{c})\left(C^{\cdot}, C^{\cdot}[1]\right)\right)<\infty$, then $F_{1}$ has a hull; in other words, $C$ has a miniversal homotopy deformation.

Proof. For $R \in \mathbf{C}, R \longrightarrow k$ has a nilpotent kernel hence the natural bijections $\operatorname{Sk}\left(L_{F_{R}}\left(d_{0} \mid C_{0}\right)\right) \longrightarrow \operatorname{Sk}\left(L_{K\left(F_{R}\right)}\left(C_{0}^{\cdot}\right)\right)$ follow by induction from Proposition 4.3 The remainder of the statement follows from Proposition 7.1

7.3. Derived deformations. Let $\mathcal{C}$ be a fixed small flat abelian $k$-linear category. For $R \in \mathbf{C}$, we consider the trivial abelian deformation $\mathcal{C} \longrightarrow \mathcal{C}_{R}: C \longmapsto(C, R \longrightarrow$ $k \longrightarrow \mathcal{C}(C, C))$ of $\mathcal{C}$ and its right adjoint $\operatorname{Hom}_{R}(k,-): \mathcal{C}_{R} \longrightarrow\left(\mathcal{C}_{R}\right)_{k} \cong \mathcal{C}$. We consider

$$
R \operatorname{Hom}_{R}^{\mathrm{res}}(k,-): D_{\mathrm{fcd}}^{+}\left(\mathcal{C}_{R}\right) \longrightarrow D_{\text {fcd }}^{+}(\mathcal{C})
$$

as in 6.3 For a fixed $C \in D_{\text {fcd }}^{+}(\mathcal{C})$, we will call the objects of $L_{R \mathrm{Hom}_{R}^{\mathrm{res}}(k,-)}\left(C^{\cdot}\right)$ derived $(R$-) deformations of $C$. We consider

$$
F_{2}: \mathbf{C} \longrightarrow \operatorname{Set}: R \longmapsto \operatorname{Sk}\left(L_{R \operatorname{Hom}_{R}^{\mathrm{res}}(k,-)}\left(C^{\cdot}\right)\right) .
$$

Proposition 7.4. If $\operatorname{dim}_{k}\left(\operatorname{Ext}_{\mathcal{C}}^{1}\left(C^{\cdot}, C^{\cdot}\right)\right) \leq \infty$, then $F_{2}$ has a hull; in other words, $C$ has a miniversal derived deformation.

Remark 7.5. In exactly the same way, the functor describing derived deformations of bounded coflat dimension and the functor describing coflat deformations of objects have a hull. The latter is shown in [1, Prop. E1.11] (for noetherian objects).

Proof. Consider $\operatorname{Hom}_{R}(k,-): \operatorname{lnj}\left(\operatorname{Ind} \mathcal{C}_{R}\right) \longrightarrow \operatorname{Inj}(\operatorname{Ind} \mathcal{C})$ and let $E$ be an injective resolution in IndC of $C$. By induction on the nilpotent kernel of $R \longrightarrow k$, Propositions 6.56.9 yield a natural isomorphism $F_{3} \cong F_{2}$ for

$$
F_{3}: \mathbf{C} \longrightarrow \text { Set }: R \longmapsto \operatorname{Sk}\left(L_{K\left(\operatorname{Hom}_{R}(k,-)\right)}\left(E^{\cdot}\right)\right) \text {. }
$$

By Lemma 7.6 below, we have $\operatorname{Inj}\left(\operatorname{Ind} \mathcal{C}_{R}\right) \cong \operatorname{Inj}\left((\operatorname{Ind} \mathcal{C})_{R}\right) \cong R \otimes_{k} \operatorname{Inj}(\operatorname{Ind} \mathcal{C})$ hence the results follows from Proposition 7.3

Lemma 7.6. For an abelian $k$-linear category $\mathcal{C}$ with enough injectives, the functor $\operatorname{Hom}_{k}(R,-): \mathcal{C} \longrightarrow \mathcal{C}_{R}$ induces an isomorphism

$$
R \otimes_{k} \operatorname{lnj}(\mathcal{C}) \cong \operatorname{lnj}\left(\mathcal{C}_{R}\right) .
$$

Proof. The composition of forgetful functors $\mathcal{C} \cong\left(\mathcal{C}_{R}\right)_{k} \longrightarrow \mathcal{C}_{R} \longrightarrow \mathcal{C}$ is (naturally isomorphic to) the identity, hence the same holds for the composition of right 
adjoints

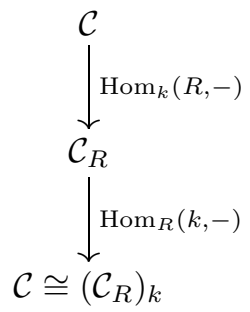

Consequently, for an injective $E \in \mathcal{C}, \operatorname{Hom}_{k}(R, E) \in \mathcal{C}_{R}$ is the unique (up to isomorphism) lift of $E$ along $\operatorname{Hom}_{R}(k,-)$ (see Theorem 6.11). For injectives $E, F \in$ $\mathcal{C}$, we have

$$
R \otimes_{k} \mathcal{C}(E, F) \cong \mathcal{C}\left(\operatorname{Hom}_{k}(R, E), F\right) \cong \mathcal{C}_{R}\left(\operatorname{Hom}_{k}(R, E), \operatorname{Hom}_{k}(R, E)\right)
$$

whence the result follows.

\section{REFERENCES}

[1] M. Artin and J. J. Zhang, Abstract Hilbert schemes, Algebr. Represent. Theory 4 (2001), no. 4, 305-394.

[2] F. M. Bleher and T. Chinburg, Deformations and derived categories, C. R. Math. Acad. Sci. Paris 334 (2002), no. 2, 97-100.

[3] A. Bondal, Representations of associative algebras and coherent sheaves, Math. USSR-Izv. 34 (1990), no. 1, 23-42.

[4] A. Bondal and A. Polishchuk, Homological properties of associative algebras: the method of helices, Russian Acad. Sci. Izv. Math 42 (1994), 219-260.

[5] P. Deligne, La conjecture de Weil II, Inst. Hautes Études Sci. Publ. Math. 52 (1980), 137-252.

[6] J. Huebschmann and T. Kadeishvili, Small models for chain algebras, Math Z. 207 (1991), $245-280$.

[7] M. Inaba, Toward a definition of moduli of complexes of coherent sheaves on a projective scheme, J. Math. Kyoto Univ. 42 (2002), no. 2, 317-329.

[8] O. A. Laudal, Formal moduli of algebraic structures, Lecture Notes in Mathematics, vol. 754, Springer-Verlag, 1979.

[9] W. T. Lowen and M. Van den Bergh, Deformation theory of abelian categories, Preprint math.CT/0405226 2003.

[10] - Hochschild cohomology of abelian categories and ringed spaces, Preprint math.KT/0405227 2004.

[11] M. Markl, Ideal perturbation lemma, Comm. Algebra 29(11) (2001), 5209-5232.

[12] M. Schlessinger, Functors of Artin rings, Trans. Amer. Math. Soc. 130 (1968), 208-222.

[13] A. Schwarz, Noncommutative supergeometry, duality and deformations, Nuclear Phys. B 650 (2003), no. 3, 475-496.

[14] M. Van den Bergh, Non-commutative quadrics, in preparation.

[15] - Non-commutative $\mathbb{P}^{1}$-bundles over commutative schemes, Preprint math.RA/0102005 2001.

[16] A. Vistoli, The deformation theory of local complete intersections, Preprint alg-geom/9703008 1997.

Departement DWis, Vrije Universiteit Brussel, Pleinlaan 2, 1050 Brussel, Belgium E-mail address, Wendy T. Lowen: wlowen@vub.ac.be 